\title{
Transcriptome Analysis of Hamelia patens (Rubiaceae) Anthers Reveals Candidate Genes for Tapetum and Pollen Wall Development
}

\author{
Lin Yue ${ }^{1,2}$, David Twell ${ }^{3}$, Yanfeng Kuang ${ }^{1 *}$, Jingping Liao' ${ }^{1}$ and Xianqiang Zhou ${ }^{4}$ \\ ${ }^{1}$ Key Laboratory of Plant Resources Conservation and Sustainable Utilization, South China Botanical Garden, Chinese \\ Academy of Sciences, Guangzhou, China, ${ }^{2}$ College of Life Sciences, University of Chinese Academy of Sciences, Beijing, \\ China, ${ }^{3}$ Department of Genetics, University of Leicester, Leicester, UK, ${ }^{4}$ Beijing Genomic Institute, Shenzhen, China
}

\section{OPEN ACCESS}

Edited by:

Dazhong Dave Zhao, University of Wisconsin-Milwaukee,

USA

Reviewed by:

Zhao Su,

Pennsylvania State University, USA Mingli XU,

University of Pennsylvania, USA

*Correspondence: Yanfeng Kuang

kuangyf05@scbg.ac.cn

Specialty section:

This article was submitted to Plant Evolution and Development, a section of the journal

Frontiers in Plant Science

Received: 28 July 2016 Accepted: 15 December 2016 Published: 06 January 2017

Citation:

Yue L, Twell D, Kuang Y, Liao J and Zhou X (2017) Transcriptome Analysis of Hamelia patens (Rubiaceae) Anthers Reveals Candidate Genes for Tapetum and Pollen Wall Development.

Front. Plant Sci. 7:1991. doi: 10.3389/fpls.2016.01991
Studies of the anther transcriptome on non-model plants without a known genome are surprisingly scarce. RNA-Seq and digital gene expression (DGE) profiling provides a comprehensive approach to identify candidate genes contributing to developmental processes in non-model species. Here we built a transcriptome library of developing anthers of Hamelia patens and analyzed DGE profiles from each stage to identify genes that regulate tapetum and pollen development. In total 7,720 putative differentially expressed genes across four anther stages were identified. The number of putative stage-specific genes was: 776 at microspore mother cell stage, 807 at tetrad stage, 322 at uninucleate microspore stage, and the highest number $(1,864)$ at bicellular pollen stage. GO enrichment analysis revealed 243 differentially expressed and 108 stage-specific genes that are potentially related to tapetum development, sporopollenin synthesis, and pollen wall. The number of expressed genes, their function and expression profiles were all significantly correlated with anther developmental processes. Overall comparisons of anther and pollen transcriptomes with those of rice and Arabidopsis together with the expression profiles of homologs of known antherexpressed genes, revealed conserved patterns and also divergence. The divergence may reflect taxon-specific differences in gene expression, the use RNA-seq as a more sensitive methodology, variation in tissue composition and sampling strategies. Given the lack of genomic sequence, this study succeeded in assigning putative identity to a significant proportion of anther-expressed genes and genes relevant to tapetum and pollen development in $\mathrm{H}$. patens. The anther transcriptome revealed a molecular distinction between developmental stages, serving as a resource to unravel the functions of genes involved in anther development in $\mathrm{H}$. patens and informing the analysis of other members of the Rubiaceae.

Keywords: transcriptome, Rubiaceae, Hamelia patens, anther, tapetum, pollen wall

\footnotetext{
Abbreviations: BCP, bicellular pollen; Ca, callose; Co, columellae; Ep, epidermis; Ec, endothecium; Ene, endexine; FL, foot layer; FT, fibrous thickenings; GN, generative nucleus; GPG, germinated pollen grain; In, intine; LD, lipid droplets; ML, middle layer; MMC, microspore mother cell; MPG, mature pollen grain; Ms, microspores; MSA: mixed stage anther transcriptome library; N, nucleus; Nu, nucleolus; PG, pollen grains; PO, protruding oncus; PrE, primexine; PS, pericytoplasmic space; RER, rough endoplasmic reticulum; RPKM, reads per kilo bases per million mapped reads; T, tapetum; TCP, tricellular pollen; TET, tetrad; Te, tectum; Tet, tetrads; UNM, uninucleate microspore; V, vacuole; VN, vegetative nucleus.
} 


\section{INTRODUCTION}

Pollen grains are the microgametophytes of seed plants that produce the male gametes required for sexual reproduction (Borg and Twell, 2011). Although the overall structure of pollen is conserved in angiosperms, pollen shows considerable variation in size, shape, and surface characteristics (HeslopHarrison, 1968; Blackmore et al., 2007). Recently, the diversity and evolution of palynological characters were documented across the angiosperms based on the most comprehensive classification APG III (2009). Many characters, such as exine structure and aperture features, have indicated key evolutionary transitions in pollen morphology and have proven to be informative at different taxonomic levels (Lu et al., 2015; Wortley et al., 2015). However, little is known about the genetic basis underlying pollen evolution. Comparative analysis has indicated significant conservation in anther gene expression patterns between Arabidopsis and maize (Ma et al., 2008). Further comparative analysis of anther and pollen transcriptome profiles for diverse angiosperms would complement understanding the conservation of the molecular mechanisms underlying pollen development (Wilson and Zhang, 2009; Rutley and Twell, 2015).

Pollen development involves the coordination of cellular activities and the underlying gene expression in sporophytic and gametophytic cells of the anther (Ariizumi and Toriyama, 2011). Anthers are composed of developing gametophytes and a number of surrounding cell layers: the innermost tapetum, the ML, the endothecium, and the outer epidermis. Initially, archesporial cells form in the L2 layer of the anther primordium and divide periclinally to form outer primary parietal cells (PPCs) and inner primary sporogenous cells. The primary sporogenous cells undergo divisions to form MMCs, whereas the PPCs undergo a series of divisions to form the cell layers of the anther wall (Scott et al., 2004). The MMCs undergo meiosis producing tetrads of haploid microspores at tetrad stage (TET). The tetrads are usually separated as free microspores after the callosic walls are dissolved by callase at the UNM stage. The free microspores divide asymmetrically to segregate the male germline and develop further into PG before release at BCP stage or TCP stage (McCormick, 1993; Twell, 2011). Pollen development relies on the complex interactions between reproductive and nonreproductive tissues of the anther, especially the tapetum, which provides enzymes for the dissolution of tetrads, nutrients to the developing microspores and materials deposited onto the pollen exine (Goldberg et al., 1993; Tsuchiya et al., 1994; Li et al., 2006).

A significant number of key genes required for anther and pollen development have been identified by forward genetic screens in model and crop plants. These genes, which include a significant number of transcriptional regulators, control formation, and degeneration of the tapetum, microspore release from the tetrad and formation of the complex pollen wall (Boavida et al., 2005; Wilson and Zhang, 2009; Shi et al., 2015). Key genes involved in tapetum formation and differentiation include, EXCESS MICROSPOROCYTES1/EXTRA SPOROGENOUS CELLS (EMS1/EXS) (Zhao et al., 2002) and TAPETUM DETERMINANT 1(TPD1) (Yang et al., 2003). Genes involved in the programmed cell death (PCD) of tapetum cells include TAZ1 (Kapoor et al., 2002), TAPETUM DEGENERATION RETARDATION (TDR) (Li et al., 2006), PERSISTENT TAPETAL CELL 1 (PTC1), API5 (Li et al., 2011), Osc6 (Zhang et al., 2010), and CEP1 (Zhang D.D. et al., 2014). The absence of these genes results in abnormal tapetal PCD and sterile pollen.

Transcriptional regulation is a major mechanism controlling anther development in Arabidopsis. The bHLH transcription factor (TF) DYSFUNCTIONAL TAPETUM1 (DYT1) plays a critical role in regulating tapetum function and pollen development (Zhang et al., 2006; Feng et al., 2012; Zhu et al., 2015). DYT1 regulates the expression of the ABORTED MICROSPORES (AMS) (Sorensen et al., 2003; Xu et al., 2014), MALE STERILITY1 (MS1) (Vizcay-Barrena and Wilson, 2006), and other tapetum preferential genes, primarily via DEFECTIVE IN TAPETAL DEVELOPMENT AND FUNCTION 1 (TDF1) (Gu et al., 2014). Important regulators of tetrad callose degradation include the Arabidopsis TF gene AtMYB103 (Higginson et al., 2003; Zhang et al., 2007), and the rice $\beta$-1,3-glucanase encoded by Osg1 (Wan et al., 2011). Key genes required for pollen exine development have also been characterized, including MS2 (Aarts et al., 1997), ACOS5 (Souza et al., 2009), CYP703A2 (Morant et al., 2007), CYP703A3 (Aya et al., 2009), and EFD (Hu et al., 2014). Moreover, recent co-expression analysis revealed that AMS acts as a master regulator coordinating pollen wall development and sporopollenin biosynthesis in Arabidopsis (Xu et al., 2014). Thus, current understanding of anther regulatory genes is overwhelmingly derived from studies of model plants and crops and little information about gene activities in developing anthers is known from non-model species.

Transcriptome analyses have significantly enriched knowledge of the repertoire of genes expressed during pollen development. Genome-scale analyses have been described for developing anthers and pollen, germinating pollen and pollen tubes, and mature pollen transcriptome data for at least 10 different species (Huang et al., 2011; Rutley and Twell, 2015). Transcriptome profiling at different developmental stages have been reported for Arabidopsis (Honys and Twell, 2004), rice (Wei et al., 2010), and tobacco (Bokvaj et al., 2015). Transcriptome studies of developing Arabidopsis anthers and pollen have revealed genes that function as early as meiosis, in the tapetum and in exine formation (Dukowic-Schulze and Chen, 2014). Recently, genome-wide coexpression analysis revealed 98 candidate genes closely associated with pollen wall development (Xu et al., 2014). Moreover, comparative studies in rice involving microarray studies of developing anthers have also revealed complex transcriptomes (Deveshwar et al., 2011).

RNA-Seq based methods have led to a dramatic acceleration in gene discovery (Barrero et al., 2011; Garg et al., 2011; Shi et al., 2011), which has rapidly broadened understanding of the complexity of gene networks and their regulation (Wang et al., 2010; Hua et al., 2011). Moreover, there are limitations of microarray technology such as lack of quantitative detection of transcripts with different probe sets, low abundance transcripts are difficult to detect, and a reference genome and/or expressed sequence tags (ESTs) are required (Huang et al., 2011; Zhao et al., 2014). Studies of anther transcriptomes based on RNA-seq 
are limited to those in a few crops and model plants, however, expression profiling of developing anthers by Next Generation Sequencing (NGS) from species whose genomes have not been sequenced is anticipated (Wang et al., 2009; Huang et al., 2011).

Rubiaceae, commonly known as the coffee family, is the fourth largest in the angiosperms and has important economic utilities; in addition to the valuable beverage crop coffee, as a source of quinine, dyes, and ornamentals. It is a eurypalynous family with great diversity in pollen size and shape, aperture type, number and position, pollen wall stratification, and sexine ornamentation (Dessein et al., 2005). Some pollen features have significant value in stimulating further palynological investigations, for example, the development of Ubisch bodies provides a potential model for studying sporopollenin deposition (Dessein et al., 2005). However, there is limited information about the gene expression patterns underlying anther and/or pollen development in Rubiaceae. Current molecular data from model species and a few crop plants are insufficient to resolve the mechanisms that regulate the development, function, and evolution of angiosperm pollen. More attention to such a palynologically well-documented family would help clarify the molecular processes underlying anther/pollen development in angiosperms and the associated evo-devo mechanisms.

In this paper, Hamelia patens Jacq. was chosen for study as a member of the coffee family. $H$. patens originates in tropical America and is a popular ornamental plant in south and southwest China. The ease of cultivation and long blooming period from May to October enables facile collection of floral material. Further, this species produces triaperturate PG, representing the plesiomorphic condition in the Rubiaceae family. These advantages make $H$. patens a potentially valuable system for developmental and molecular research in Rubiaceae.

Transcriptome profiles of Hamelia patens developing anthers from MMC to BCP grain stages were investigated using NGS and NGS-based digital gene expression (DGE) tag profiling. The aims of the present study were, (1) to provide molecular insight into anther and pollen development of this phylogenetically and palynologically important family, and (2), to reveal novel candidate genes involved in tapetum and pollen development, to shed light on the evo-devo mechanisms of angiosperm pollen development.

\section{MATERIALS AND METHODS}

\section{Sample Preparation for Anther Development}

Flowers of Hamelia patens Jacq. at various developmental stages were harvested from plants growing in South China Botanical Garden (SCBG), Guangdong Province, China. Each flower contains five anthers. The length of flowers and the anthers was measured using a stereo microscope. The classification of flowers and anthers for categorization of anther developmental stages is summarized in Table $\mathbf{1}$.

The anthers were fixed in $2.5 \%$ glutaraldehyde in $0.1 \mathrm{~mol} / \mathrm{L}$ phosphate buffer, $\mathrm{pH} 7.2$, placed under a vacuum for $2 \mathrm{~h}$, then stored at $4^{\circ} \mathrm{C}$ for several days. After removal from storage, the
TABLE 1 | Classification of flowers and anthers for categorization of anther developmental stages.

\begin{tabular}{lcc}
\hline Pollen developmental stage & Flower length $(\mathbf{c m})$ & Anther length (cm) \\
\hline Microspore mother cell stage & $0.30-0.80$ & $0.20-0.45$ \\
Tetrad stage & $0.90-1.00$ & $0.50-0.60$ \\
Uninucleate microspore stage & $1.10-1.60$ & $0.60-0.85$ \\
Bicellular pollen stage & $1.80-2.70$ & $0.91-1.01$ \\
\hline
\end{tabular}

anthers were rinsed in $0.1 \mathrm{~mol} / \mathrm{L}$ phosphate buffer for $2 \mathrm{~h}$, and post-fixed in $1 \%$ osmium tetroxide overnight. Following postfixation, anthers were washed in phosphate buffer, dehydrated in an acetone series, embedded in PON-812 resin and cured at $70^{\circ} \mathrm{C}$. Semi-thin sections $(1-2 \mu \mathrm{m})$ were cut with glass knives on a LKB-11800 microtome, stained with $0.1 \%$ Toluidine Blue, and observed and photographed with a Leica - DM5500B light microscope (LeiCa, Germany). Ultrathin sections (80 nm) were cut using a Leica-Ultracut $S$ ultramicrotome with a diamond knife, and stained with uranyl acetate and lead citrate. Transmission electron micrographs were taken with a JEOL JEM1010 (JEOL, Japan) transmission electron microscope at $100 \mathrm{KV}$.

For SEM, the PG were mounted on copper stubs with a strip of double-sided conductive tape and air-dried. The sample was then coated with gold in a JEOL JFC-1600 sputter coater. Observations and digital images were collected with a JEOL JSM-6360LV SEM. Measurements of the polar axis (P) and equatorial diameter (E) were made on digital SEM images with JEOL's Smile View software.

\section{Sample Preparation for RNA Extraction}

Transcriptome profiling was carried out on developing anthers at four landmark stages: MMC stage, tetrad (TET) stage, UNM stage, and BCP stage. The four stages were defined on the basis of cytological observations with LM and TEM as described above and confirmed using DAPI staining.

Fresh flowers were collected from three well-managed populations of $H$. patens in the SCBG. The anthers were picked out under a dissecting microscope, immediately frozen in liquid nitrogen and stored at $-80^{\circ} \mathrm{C}$ until RNA extraction. They were classified into four separate groups according to length and each group was pooled by mixing equal quantities of anthers from the three populations.

Total RNA was extracted using pBIOZOL method. RNA quality was characterized by using a NanoDrop ND1000 spectrophotometer (NanoDrop, USA), and by determination of the RIN (RNA Integrity Number) value ( $\geq 7.3$ ) using an Agilent 2100 Bioanalyzer.

\section{Library Preparation and Sequencing}

The cDNA libraries were prepared following manufacturer's instructions (Illumina, USA). Five cDNA libraries (MMC, TET, UNM, BCP, and MSA) were established. MSA was a mixed stage anther sample containing equal amounts of anthers from each of the four stages (MMC, TET, UNM, and BCP). mRNA was enriched using oligo (dT) magnetic beads from $5 \mu \mathrm{g}$ total RNA (total RNA amount of MSA was $20 \mu \mathrm{g}$ ). To avoid priming bias 
during cDNA synthesis, isolated mRNAs were first fragmented into short pieces (about $200 \mathrm{bp}$ ) using RNA Fragmentation Reagents (Ambion, USA). The cleaved mRNA fragments were converted to double-stranded cDNA using random hexamer primers (Illumina) with the SuperScript Double-Stranded cDNA Synthesis kit (Invitrogen, USA). The double-stranded cDNA was purified using a QiaQuick PCR Purification kit (Qiagen, USA) and was processed by end-repair using End-Repair Mix Reaction System (Beijing Genomic Institute, China) and the addition of a single adenine. The repaired cDNA fragments were then ligated with sequencing adapters. To select a size range of templates for downstream enrichment, the products of the ligation reaction were purified on a $2 \%$ TAE-agarose gel. Then the purified cDNA was enriched by PCR amplification using Primer PE 1.0 and PE 2.0 (Illumina) complementary to the ends of the adapters with Phusion DNA Polymerase. The library products were then sequenced using Illumina HiSeq ${ }^{\circledR} 2000$ at the Beijing Genomic Institute, China.

\section{Data processing and De novo Assembly}

The original data produced by the sequencer were defined as raw reads. Base calling accuracy was measured by the Phred quality score (Q score), which is the most common metric used to assess the accuracy of a sequencing platform. Q20 stands for $99 \%$ accuracy representing probability of incorrect base call being 1 in 100, while Q30 stands for 99.9\% accuracy representing probability of incorrect base call being 1 in 1000 (Illumina technique notes: sequencing). Filtering of raw reads was carried out to obtain clean reads by remove those, (a) containing adaptors, (b) containing more than 5\% unknown nucleotides, and (c) showing more than $10 \%$ of bases with a Phred scaled probability (Q) less than 20.

Clean reads were assembled into unigenes using the short reads assembly program Trinity, release-20130225 ${ }^{1}$. The resulting unigenes were divided into two major classes. One class containing unigenes that had a similarity of more than $70 \%$, and a singletons class (Iseli et al., 1999; Grabherr et al., 2011).

\section{Functional Annotation and Analysis}

To assign putative gene function, unigene sequences were firstly aligned by BLASTx $(E$-value $<1 \mathrm{e}-5)$ to protein databases (NR, SwissProt, KEGG, COG). Then the unigenes were searched against nucleotide database NT $(E$-value $<1 \mathrm{e}-5)$ using BLASTx to retrieve proteins with the highest sequence similarity with the given unigenes and their protein functional annotations. Functional categories of the predicted genes were obtained by applying gene ontology (GO) terms to the NR database annotation using the Blast2GO program. GO functional classification for all unigenes and the distribution of gene functions were analyzed using WEGO software (Conesa et al., 2005; Ye et al., 2006). To identify possible functions, the annotated unigene sequences were searched in the COG database where orthologous gene products are classified (Kanehisa et al., 2008). To identify active pathways in $H$. patens anthers, annotated sequences were mapped to reference pathways in the KEGG

\footnotetext{
${ }^{1}$ http://trinityrnaseq.sourceforge.net/
}

database (Release 63.0). COG and KEGG pathway annotations were performed using BLASTall software.

\section{Comparison with the Coffee Genome}

Since Coffea canephora is the only species in the Rubiaceae with a sequenced genome, comparison analysis was conducted between the unigenes of $H$. patens and the $C$. canephora CDSs using BLASTn. Sequence similarity was estimated by the coverage of the matched length, by calculating the value of matched sequence length versus unigene length and CDS length, respectively. The coffee genome was downloaded from the following website ${ }^{2}$. Moreover, the expression level and the GO annotations were examined on a set of genes with relatively high sequence similarity to their orthologs in coffee.

\section{Digital Gene Expression Tag Profiling Analysis}

In order to predict putative genes correlating with tapetum and pollen development, four separate DGE libraries (MMC, TET, UNM, and BCP) were established as mentioned above and analyzed. Procedures of library construction and sequencing were the same with the MSA library. All clean reads from the four libraries were mapped onto the transcriptome library (MSA) to calculate the expression level for each gene and the gene coverage using SOAP (version 2.21). If there was more than one transcript for a gene, the longest one was used to calculate its expression level and coverage. The gene expression level was calculated using RPKM value. The gene coverage was determined as the ratio of the base number in a gene covered by unique mapping reads to the total bases number of the gene. RPKM value $\geq 10$ was used as a criterion to define high-level expressed genes. RPKM value of 10 is the average of the median for every gene plus 5. Highly expressed genes were categorized with a Venn diagram.

Digital Gene Expression was used to compare the differences in gene expression (Audic and Claverie, 1997). The threshold FDR $<0.001$ and the absolute value of the $\log _{2}$ Ratio $\geq 1$ were used to determine the potential difference in gene expression. Genes enriched at a specific stage were identified by comparing gene expression between two adjacent stages. Genes that were twofold up-regulated and expressed at high-level $(\mathrm{RPKM} \geq 10)$ at the same stage were identified. Differentially expressed genes (DEGs) were twofold up-regulated at only one stage or simultaneously at two stages or three stages, which were identified by pairwise comparison of the four stages of anther development. Among the twofold up-regulated genes at only one stage, those with high expression (RPKM value $\geq 10$ ) at the same stage and meanwhile with low expression (RPKM value $\leq 10)$ at the other three stages were defined as specifically expressed genes (SEGs). Then we carried out GO functional enrichment and KEGG pathway enrichment analysis for these SEGs. Cluster analysis of gene expression patterns was performed with Cluster (De Hoon et al., 2004) and Java Treeview (Saldanha, 2004) softwares. With GO enrichment analysis, first all DEGs were mapped to GO terms in the database ${ }^{3}$, then the gene numbers

\footnotetext{
${ }^{2}$ http://coffee-genome.org/download

${ }^{3}$ http://www.geneontology.org/
} 
for every term was calculated, hypergeometric test was used to find significantly enriched GO terms in DEGs. The calculated $p$-value went through Bonferroni Correction, taking corrected $p$-value $\leq 0.05$ as a threshold.

With $\mathrm{Nr}$ annotation, Blast2GO program was used to get GO annotation of DEGs, and then WEGO software (Ye et al., 2006) was used to do GO functional classification for DEGs. KEGG pathway enrichment analysis was used to identify significantly enriched metabolic pathways or signal transduction pathways in DEGs (Kanehisa et al., 2008).

\section{Real-Time Quantitative PCR (q-PCR)}

qRT-PCR was performed on independently collected samples to verify the potentially differentially expressed genes (DEGs) identified by RNA-seq. Total RNA was prepared from $H$. patens anthers at the four developmental stages as the same for DGE analysis. First-strand cDNA was synthesized using a FastQuant RT Kit (TIANGEN, China) according to the manufacturer's protocol. Real-time PCR primer was designed on Web Primer: DNA and Purpose Entry website ${ }^{4}$. Primers used in the experiment are listed in Supplementary Table S1. All reactions were performed using GoTaq qPCR Master Mix (Promega, USA). Reactions were carried out in a total volume of $10 \mu \mathrm{L}$ reaction mixture containing $5.0 \mu \mathrm{L}$ of GoTaq qPCR Master Mix (Promega), $0.2 \mu \mathrm{L}(10 \mu \mathrm{mol} / \mathrm{L})$ of each primer, $200 \mathrm{ng}$ of template cDNA. The real-time RT-PCR amplification was performed with LightCycler 480|| Real-Time PCR System using two-step cycling conditions of $95^{\circ} \mathrm{C}$ for $10 \mathrm{~min}$, followed by 40 cycles of $95^{\circ} \mathrm{C}$ for $15 \mathrm{~s}$ and $60^{\circ} \mathrm{C}$ for $60 \mathrm{~s}$. Dissociation stage condition was set at $95^{\circ} \mathrm{C}$ for $15 \mathrm{~s}, 60^{\circ} \mathrm{C}$ for $15 \mathrm{~s}$, and $95^{\circ} \mathrm{C}$ for $15 \mathrm{~s}$. The $\beta$-Actin primer was designed based on the homologous gene of Rubia cordifolia L. $\beta$-Actin was amplified from $H$. patens anthers at the same four stages and used as an internal control. The relative quantities of transcripts were calculated using the comparative Ct method and three biological replicates were performed.

\section{RESULTS}

\section{Ultrastructural Observations of Anther Wall and Pollen Development}

Anthers of $H$. patens contain four microsporangia. In cross sections, the MMCs are angular in shape and possess a large nucleus with a darkly staining $\mathrm{Nu}$ (Figure 1A). The anther wall at the MMC stage is differentiated into epidermis, endothecium, $\mathrm{ML}$, and tapetum. The tapetum is uninucleate, one or two layered and the MMCs become oval prior to meiosis (Figure 1A).

After meiosis, tetrads of haploid microspores fill the anther locules (Figure 1B). Tetrads are surrounded by a thick asymmetric callose envelope and the primary cell wall is visible outside of the callose. The plasmalemma of the microspores is initially straight and in direct contact with the callose (Figure 2A). Subsequently, a fibrillar surface coat develops between the callose and the plasmalemma, which has a loose, irregular fibrillar texture and is considered primexine matrix

${ }^{4} \mathrm{http}: / /$ www.yeastgenome.org/cgi-bin/web-primer

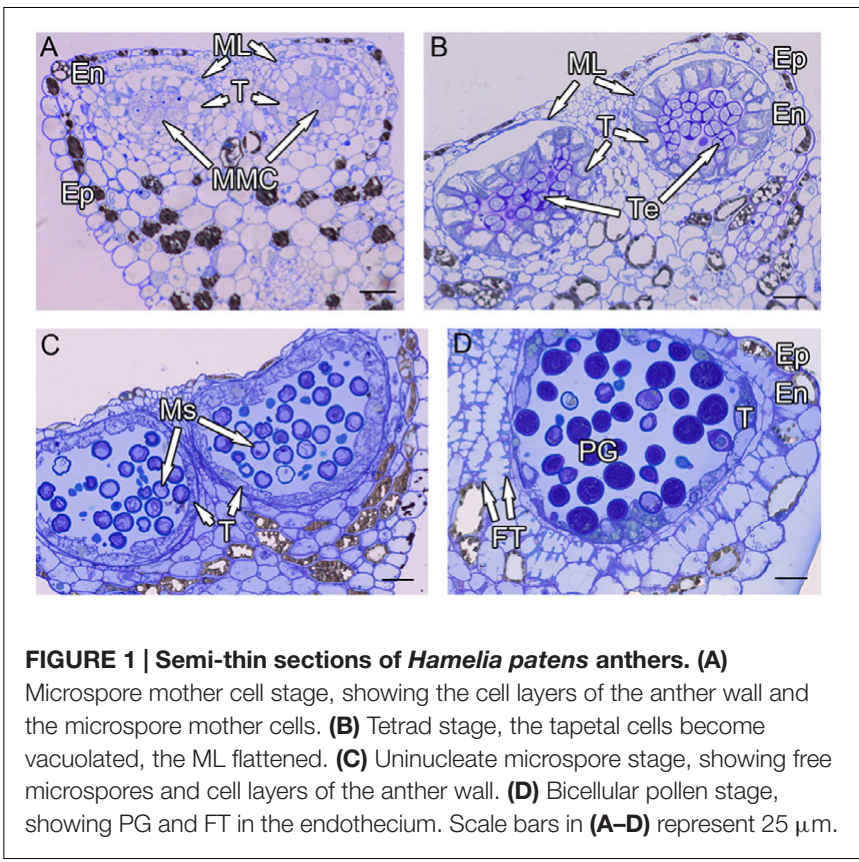

(Figure 2B). Rod-shaped electron-dense units are radially oriented in the distal part of the primexine, which eventually form the columellae. At this stage, the epidermis, endothecium, and ML become high vacuolated, nuclei are displaced to the wall and nucleoli are fragmented. The ML becomes flat (Figure 1B). Tapetal cells contain numerous ribosomes and extensive layers of endoplasmic reticulum that lie below the cell membrane and surround the nuclei in concentric rings. Clusters of small vesicles are present throughout the tapetal cytoplasm and pre-Ubisch bodies are formed (Figure 2C).

At uninucleate stage, microspores are released from the tetrads into the anther locule, and increase both in volume and in wall thickness (Figure 1C). The epidermis and endothecium are high vacuolated and contain lipidic materials and starch grains, whereas the ML is almost degenerated. Abundant Ubisch bodies are produced in the tapetal cells and extruded through their inner tangential and radial walls (Figure 2D). The Ubisch bodies possess an irregular and granular shape. The electron density of the Ubisch body wall is very similar to that of the exine. During the early development of free microspores, the columellae increase in thickness, particularly at their distal ends, the tectum develops, while the sporopollenin-like materials are deposited between the distal portions of the columellae. The FL occurs at the bases of the columellae (Figure 2E). White-line-centered lamellae were observed between the FL and the endexine (Figure 2F). The endexine continues to develop on the proximal surface of these white lines and abundant electron-dense material, probably sporopollenin, appears beneath the endexine. Subsequently, the columellae become solid and the intercolumellar space is filled with fibrillar materials, i.e., remnants of the primexine matrix. As the microspores form a large vacuole (Figure 2G), the plasmalemma in the interapertural regions recedes, creating a large PS under the radially oriented membranous granular 

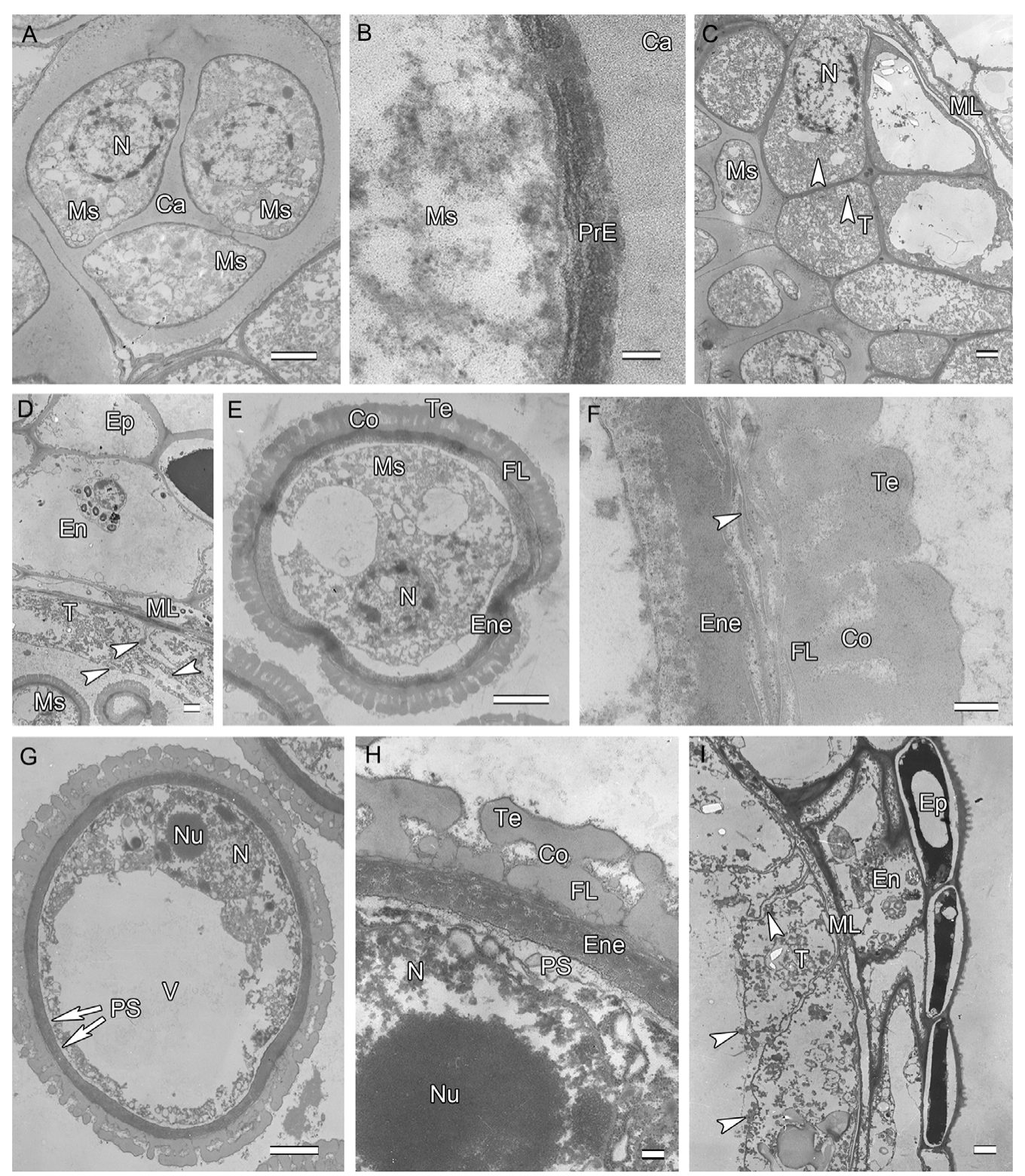

FIGURE 2 | Ultra-thin sections of $\boldsymbol{H}$. patens anthers. (A) Tetrad stage, the tetrads encased by a communal callose wall. (B) Tetrad stage, primexine matrix forms between the callose wall and the microspore. (C) Tetrad stage, showing fragmentation of nucleoli, small vesicles, and pro-Ubisch bodies (white arrowheads). (D) Uninucleate microspore stage, showing abundant Ubisch bodies produced in the tapetum (white arrowheads). (E) Showing a free microspore with differentiated wall. (F) Showing white-line-centered lamella (white arrowhead) between the FL and the endexine. (G) Vacuolated microspore stage, the nucleus is positioned against the cell wall. (H) Showing the PS formed beneath the exine. (I) Showing cell layers of the anther wall and Ubisch bodies in the tapetum (white arrowheads). Scale bars in (A,C-E,G,I) represent $2 \mu \mathrm{m}, \mathbf{( B )}$ represents $100 \mathrm{~nm}, \mathbf{( F , H )}$ represent $200 \mathrm{~nm}$.

material layer (Figure $\mathbf{2 H}$ ). This event might indicate the start of intine development. At this phase, the tapetal cell walls and cytomembranes are completely degenerated, a large number of vesicles and lipids are present throughout the tapetal cells and Ubisch bodies are released from the surface of the tapetal cells into the locule (Figure 2I). At this phase, a thin layer of cuticle is present at the anther epidermis (Figure 2I).

Initiation of intine development takes place prior to the first pollen mitosis. At this stage the intine has a fibrillar structure and microspores possess abundant organelles, e.g., RER and a large vacuole (Figure 3A). The cytoplasm contains long profiles of RER parallel with the plasmalemma and numerous mitochondria. Later the microspores enter $\mathrm{BCP}$ stage in which the generative cell is surrounded by $\mathrm{LD}$ (Figure 3B). The cytoplasm is characterized by an abundance of compound starch grains and LD (Figure 3B). The intine thickens and appears more electron-dense and reaches the endexine at several sites (Figure 3C). Initiation of the characteristic FT takes place in the endothecium (Figure 1D). 

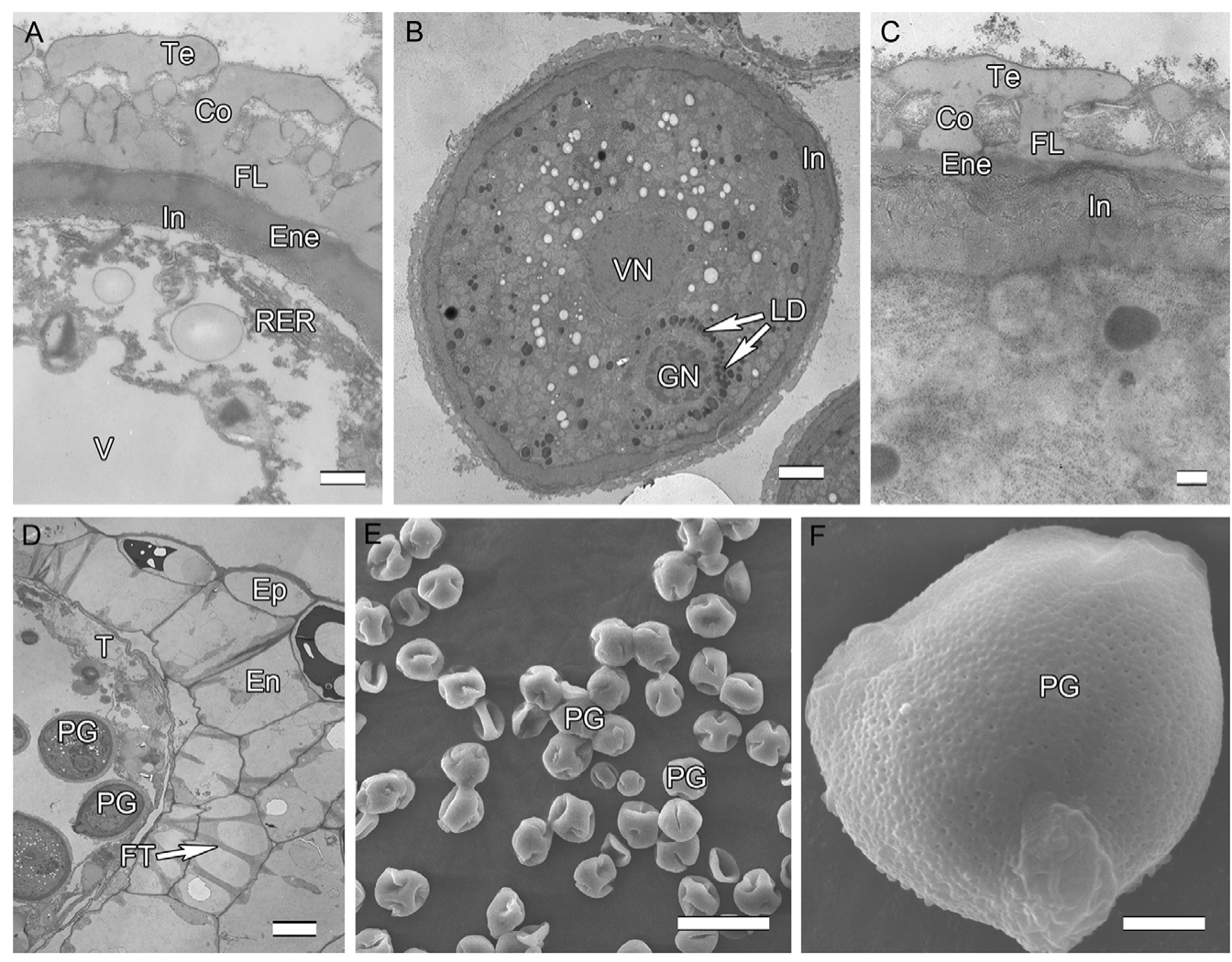

FIGURE 3 | Ultra-thin sections of $\boldsymbol{H}$. patens anthers. (A) Uninucleate microspore stage, showing the early-formed intine and associated RER. (B) Bicellular pollen stage, showing the GN surrounded by LD. (C) Bicellular pollen stage, showing the thickened and more electron-dense intine. (D) Bicellular pollen stage, showing cell layers of the anther wall, fibrous thickenings in the endothecium, and remnants of the tapetum. (E) MPGs released from the anther locule. (F) One PG, showing the micro-reticulate ornamentation and PO. Scale bars in (A) represents $500 \mathrm{~nm}$; (B) represents $2 \mu \mathrm{m}$; (C) represents $200 \mathrm{~nm}$; (D) represents $10 \mu \mathrm{m}$; (E) represents $50 \mu \mathrm{m}$; (F) represents $5 \mu \mathrm{m}$.

Cells of the tapetum and ML completely degenerate, leaving the endothecium as the innermost anther wall layer (Figure 3D). The volume of the PG progressively increases, resulting in stretching of the pollen wall, which is more pronounced in PG at dehiscence. Projection of the intine in the aperture region was observed in some PG (Figures 3D,F). Pollen grains are bicellular at anthesis, relatively small [P (polar axis) 18.36 (17.819.4) $\mu \mathrm{m} \times \mathrm{E}$ (equatorial diameter) $19.66(18.7-20.9) \mu \mathrm{m}]$, and oblate spheroidal in equatorial view (Figure 3E). The grains are planaperturate with three compound apertures. The exine pattern is micro-reticulate with the sculpting somewhat obscured by the presence of fibrillar material that fills the spaces between the columellae (Figure 3F).

\section{cDNA Sequence Generation and De novo Assembly}

Approximately 948 million bases were generated and a set of 105 million 90 bp paired-end reads were obtained after cleaning and quality checks. Q20 and Q30 values reached 97.8 and $92.1 \%$, respectively, representing high quality sequencing. Assembly of reads resulted in 177,548 contigs with an average length of $354 \mathrm{nt}$. These were assembled into 89,849 unigenes, which were divided into two classes. The first class is distinct singletons $(52,379)$ with the prefix unigene, representing unigenes derived from a single gene. The second class is distinct cluster unigenes with the prefix CL and the cluster ID, where each cluster contains unigenes with similarities of more than $70 \%$. The 37,470 cluster unigenes are distributed into 14,948 distinct clusters, among which, 3,263 contain one unigene (unigenes shorter than 200 bp were removed after family clustering), and 7,247 contain two unigenes. The remaining 4,438 clusters contain three or more unigenes, and the largest two clusters contain 45 unigenes. The cluster unigenes may be derived from the same gene or from a homologous gene (Grabherr et al., 2011).

\section{Gene Annotation and Functional Classification}

In the whole unigene set, a total of 57,476 (64\%) unigenes were significantly matched to known genes in the public databases of NR, NT, Swiss-Prot, KEGG, COG, and GO (Table 2), representing putative functional annotations for more than half of the assembled sequences.

Homology searches performed using BLASTx against the nonredundant (NR) protein database showed 55,563 (61.8\%) of unigenes with matches to the NR database with an $E$-value cut-off of $1 \mathrm{e}-5$ (Supplementary Figure S1A). The similarity distribution 
TABLE 2 | Annotation results of Hamelia patens unigenes.

\begin{tabular}{|c|c|c|c|c|c|c|c|}
\hline Sequence File & NR & NT & Swiss-Prot & KEGG & COG & GO & ALL \\
\hline H. patens-Unigene & $55,563(61.8 \%)$ & 46,601 (51.9\%) & $35,244(39.2 \%)$ & 31,169 (34.7\%) & $18,948(21.1 \%)$ & 42,244 (47.0\%) & $57,476(64.0 \%)$ \\
\hline
\end{tabular}

shows $68.6 \%$ of BLASTx hits are within the range $60-100 \%$, representing strong homology. Only $31.4 \%$ have similarity values less than $60 \%$, showing moderate homology (Supplementary Figure S1B). With regard to species similarity, the highest proportion of matched sequences in the NR database are derived from Solanum lycopersicon (33.9\%, 18,815 unigenes) and Vitis vinifera (26.4\%, 14,667 unigenes) which belong to the Solanales, a sister order to the Gentianales, to which Rubiaceae belongs (APG III, 2009; Supplementary Figure S1C). H. patens shows a similar proportion $(27.2 \%, 15,090$ unigenes) of sequences matching the genome of Coffea canephora, which was published recently (Denoeud et al., 2014; Dereeper et al., 2015). Further comparative analysis between $H$. patens and $C$. canephora is presented in section "Comparison with the Coffee Genome" below.

To understand the potential function of the assembled unigenes, the COGs (clusters of orthologous group of proteins) functional annotation system was used. In total, 18,948 (21.1\%) unigenes were mapped to the COG database and possible functions and statistics were predicted. The five largest categories are general function (17.9\%), transcription (8.9\%), replication, recombination, and repair (8.6\%), signal transduction mechanisms (7.0\%), and posttranslational modification, protein turnover, and chaperones (6.9\%) (Supplementary Figure S2). The proportion of genes annotated by COG and the predicted categories are quite similar in other studies of non-model species without known genomic sequences in the past a few years; for instance, 20.4\% in Triadica sebifera in Euphorbiaceae (Divi et al., 2016), 20.1\% in Uncaria rhynchophylla in Rubiaceae (Guo et al., 2014), and $21.1 \%$ in Dendrocalamus latiflorus in Gramineae (Zhang et al., 2012).

Gene ontology assignments were used to classify the functions of predicted genes, resulting in 42,244 (47.0\%) unigenes assigned to at least one GO term, while 47,605 (53.0\%) could not be assigned. Among the 42,244 BLASTable ( $E$-value $<1 \mathrm{e}-5$ ) unigenes, 17,923 unigenes are distinct singletons, while 24,321 are distinct cluster unigenes, distributed in 9,639 clusters. If the unigenes contained in the distinct clusters were treated as single unigenes, then the total number of annotated unigenes would be 27,562 , and the annotated proportion would be $41.0 \%$, which is close to the proportion of unigenes in the same cluster treated as independent unigenes. Non-BLASTable $(E-$ value $>1 e-5)$ sequences have been reported in all studied plant transcriptomes, with the proportion varying from 13.0 to $80.0 \%$, depending on the species, the sequencing depth and the parameters of the BLAST search (Parchman et al., 2010; Blanca et al., 2011; Ness et al., 2011; Zhang et al., 2012, Zhang F.J. et al., 2014). Non-BLASTable (E-value $>1 \mathrm{e}-5$ ) sequences might result from biological factors, including rapidly evolved genes with divergent sequences, species-specific genes, and the persistence of non-coding fractions derived mainly from untranslated regions (Logacheva et al., 2011; Zhang et al., 2012). The BLASTable
( $E$-value $\leq 1 \mathrm{e}-5)$ unigenes are divided into three GO categories: biological process $(32,700)$, cellular component $(33,751)$, and molecular function $(31,293)$. In the biological process category, the most abundant sequences are classified into cellular process $(81.0 \%)$, metabolic process $(77.0 \%)$, and single-organism process (58.4\%). In the cellular component category, cell (93.6\%), cell part (93.6\%), and organelle $(74.6 \%)$ are the three most represented GO terms. In the molecular function category, catalytic activity (66.7\%), binding (63.6\%), and transporter activity $(10.0 \%)$ are highly represented (Supplementary Figure S3). The constitution of the most represented GO terms shows a high degree of similarity with the GO annotation of C. canephora (Dereeper et al., 2015).

To identify biological pathways activated during anther development of $H$. patens, the assembled unigenes were annotated against the KEGG database $(E$-value $<1 \mathrm{e}-5)$. A total of $31,169(34.7 \%)$ unigenes were mapped into 128 pathways in the KEGG database (Supplementary Table S2). The maps with the highest unigene representation are metabolic pathways $(6,975$, $22.4 \%)$, followed by biosynthesis of secondary metabolites $(3,465$, $11.1 \%)$, plant-pathogen interaction $(1,796,5.8 \%)$, and plant hormone signal transduction $(1,609,5.2 \%)$. This constitution exhibits a high degree of similarity with rice anther transcriptome profiles derived from microarray analysis (Deveshwar et al., 2011).

\section{Comparison with the Coffee Genome}

Alignments of the $H$. patens unigenes $(89,849)$ with the coffee genome (25,574 CDSs) revealed 15,090 (16.8\%) unigenes with a significant overlap to $7,313(28.6 \%)$ coffee CDSs. This proportion is lower than expected given that both species are from the same family. When clean reads (105 million) were mapped to the coffee genome allowing a maximum $2 \mathrm{bp}$ mismatch, a total of $366,227(0.35 \%)$ reads matched 4,047 (15.8\%) coffee CDSs, a lower proportion than observed for unigene alignments. Meanwhile, the number of reads with no mismatches was 8,821 , corresponding to 286 coffee CDSs, and 79,220 reads with a $1 \mathrm{bp}$ mismatch corresponding to 1,549 coffee CDSs. It is predictable that as mismatched bases increase, more matched reads and coffee CDS will result. Read alignment is presumed to be more accurate, since the reads represent the most comprehensive data. The low proportion of matches between $H$. patens and C. canephora might be explained by the fact that these species are not genetically close and are members of distant tribes in different subfamilies.

Among the 7,313 coffee CDSs, 3,774 have just one matching unigene, while the remaining 3,539 match multiple unigenes. In these cases, most of those matching unigenes are from the same cluster and are longer than the matched coffee CDS. This is indicative of sequence similarities other than matches with different portions of the same coffee CDS. Among these 
aligned unigenes of $H$. patens, $80(0.5 \%)$ are related to anther wall and pollen wall development (Supplementary Table S3). The remaining unigenes are largely annotated with response to salt stress, DNA-dependent regulation of transcription, oxidation-reduction process, response to cadmium and protein phosphorylation, which represent general functions.

\section{Digital Gene Expression Tag Profiling}

The numbers of unigenes mapped by clean reads in each of the four libraries are 70,735 (MMC), 71,510 (TET), 71,248 (UNM), and 70,288 (BCP). Among these, 29,002 (41.0\%), 28,660 (40.8\%), $26,806(37.6 \%)$, and 26,920 (38.3\%) genes have RPKM values $\geq 5$ in MMC, TET, UNM, and BCP, respectively. Detecting low abundance transcripts is one of the advantages of RNA-seq. In this study, 14,059 (19.9\%), 13,731 (19.2\%), 15,399 (21.6\%), and $15,617(22.2 \%)$ genes are low abundance in MMC, TET, UNM, and $\mathrm{BCP}$, respectively, with $\mathrm{RPKM} \leq 1$. Genes with $\mathrm{RPKM} \geq 10$ at one or more stages were considered highly expressed and the numbers detected at MMC, TET, UNM, and BCP stages were 18,593 (26.3\%), 18,129 (25.4\%), 16,995 (23.9\%), and 17,370 (24.7\%), respectively, (Figure 4). It is supposed that unigenes with higher expression are more likely to be full-length, therefore further analyses was focused on genes with RPKM values $\geq 10$ at one or more stages. These are classified into four main categories and 15 sub-categories based on expression pattern. The four main categories are highly expressed (RPKM value $\geq 10$ ) in one, two, three, or in all four stages. The numbers of genes of each sub-category are calculated and illustrated in the Venn diagram (Figure 5). In total, 11,570 genes are highly expressed across all stages, which may suggest their involvement in housekeeping functions or general metabolism.

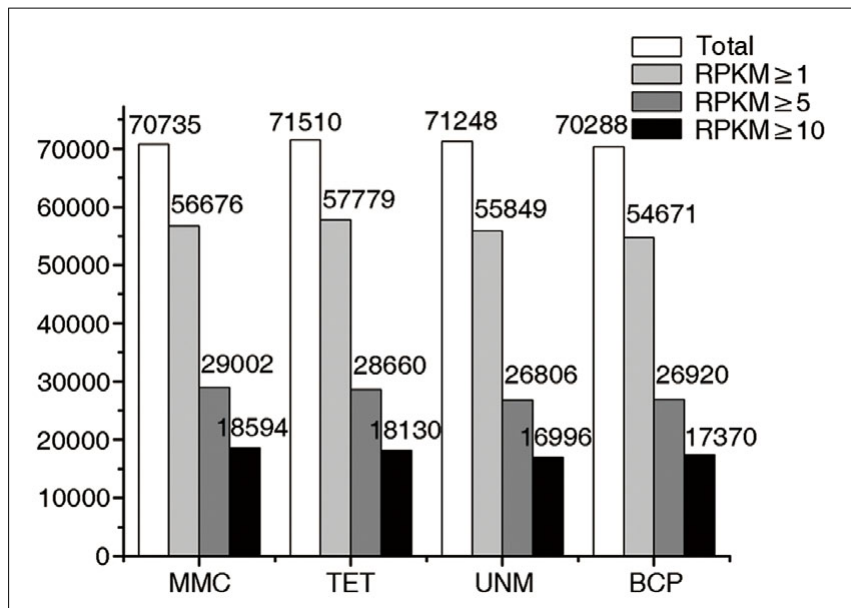

FIGURE 4 | Digital gene expression (DGE) libraries of four developmental stages. The white bars in this bar graph depict the numbers of genes mapped by clean reads in each library. The light gray bars depict the numbers of genes with RPKM value $\geq 1$ in each library. The dark gray bars depict the numbers of genes with RPKM value $\geq 5$ in each library. The black bars depict the numbers of genes with RPKM value $\geq 10$ in each library.

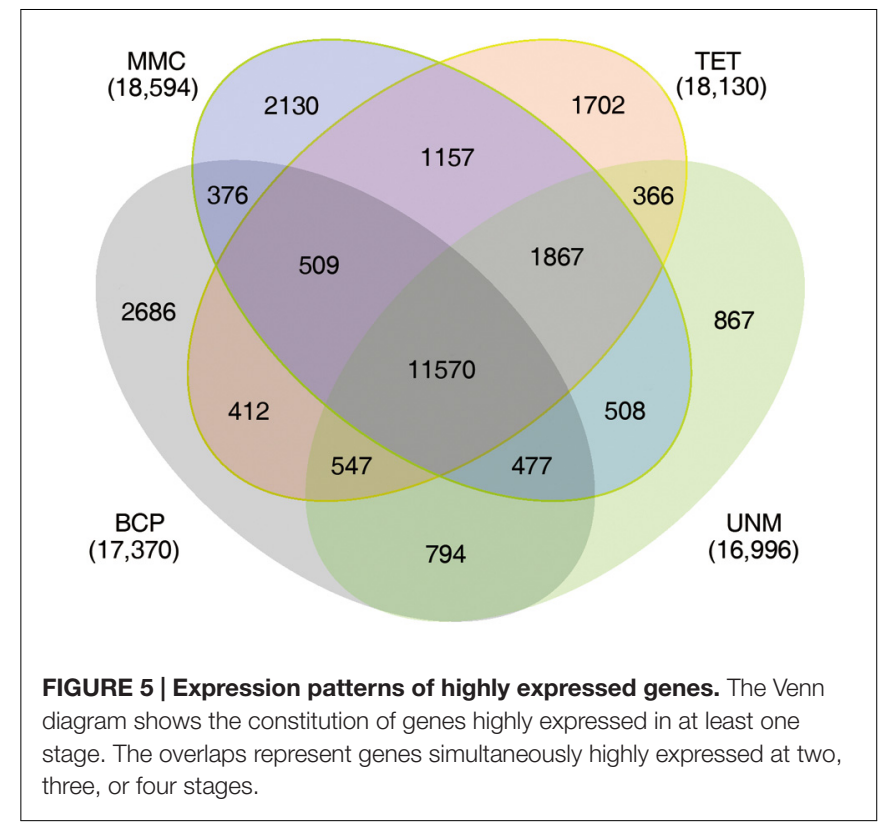

\section{Identification of Up and Down-Regulated Genes and Their GO Annotations}

The comparisons of adjacent stages, identified 2,851, 2,491, and 4,781 genes that were potentially up-regulated by at least twofold, while 3,443, 3,591, and 4,305 genes were potentially downregulated in TET, UNM, and BCP stage, respectively. A greater number of genes were down-regulated in comparison to those up-regulated in TET and UNM, however, this trend is reversed in BCP where a larger proportion of genes showed up-regulation (Figure 6). Among these putatively up-regulated genes, those with high expression $(\mathrm{RPKM}$ value $\geq 10)$ in one particular stage and lower expression (RPKM $<10)$ in the other three stages, were considered stage-enriched. 927 (32.5\%), 454 (18.2\%), and 2,068 $(43.3 \%)$ genes are enriched in TET, UNM, and BCP, respectively (Figure 6). BCP has the largest proportion of stage-enriched genes in all the stages analyzed, moreover, both up- and downregulated genes increase dramatically in BCP in comparison to earlier stages, highlighting the distinct gene expression profile at this stage.

Functional association of the potentially up-regulated and stage-enriched genes based on GO annotations (Supplementary Table S4) revealed that TET stage-enriched and up-regulated genes were enriched in protein phosphorylation, metabolic process, oxidation-reduction process, histone H3-K9 methylation, DNA methylation, DNA-dependent regulation of transcription. UNM stage-enriched and up-regulated genes were enriched in oxidation-reduction process, DNA-dependent regulation of transcription, proteolysis, metabolic process, transmembrane transport and response to cadmium ion. Categorization of BCP stage-enriched and up-regulated genes into GO functional groups showed enrichment for genes involved in oxidation-reduction process, pollen tube growth, plant-type cell wall modification, protein phosphorylation, and transmembrane transport, which could be important 


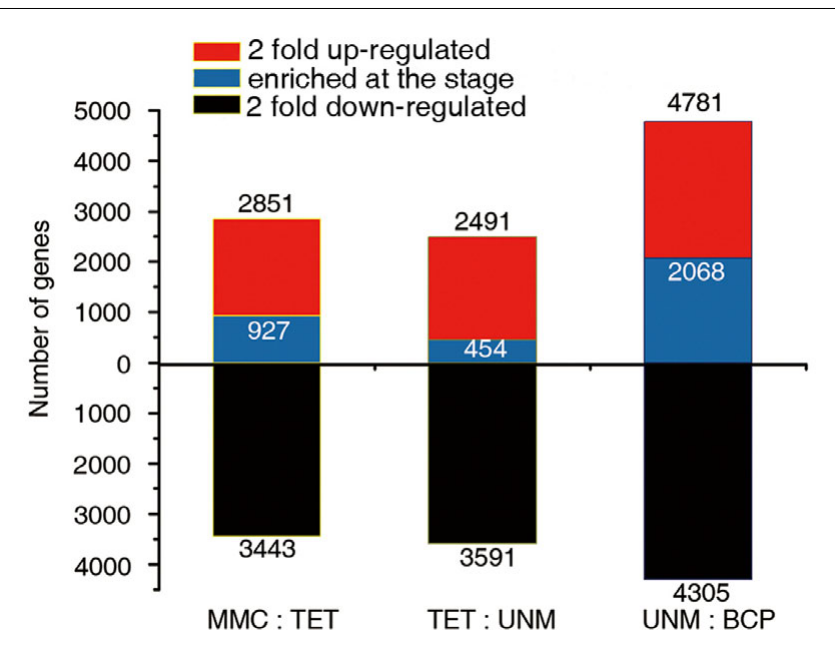

FIGURE 6 | Comparisons of adjacent developmental stages. The DGE libraries of four stages were compared to their preceding stage during anther development. MMC has no reference. The number of genes twofold up-regulated or down-regulated at FDR $\leq 0.001$ are plotted on the red or black bars in the graph. Among the up-regulated genes at each stage, the numbers of specifically enriched genes (RPKM value $\geq 10$ ) are plotted on the blue bars.

contributors to pollen maturation and the pollen transcriptome. However, a large number $(38,440)$ of BCP expressed genes have not been previously reported, which could serve as a useful resource to mine transcripts for validation of putative gametophyte and/or germline functions.

Genes with GO terms (Supplementary Table S5) related to anther wall and pollen wall development were further selected (Supplementary Figure S4). Among the putatively up-regulated genes in TET stage, $40(46.5 \%)$ are related to pollen exine development, $40(46.5 \%)$ are related to other components of, or contributing to, pollen wall development and six (7.0\%) are related to tapetum development. Among the stage-enriched genes in TET stage, six $(35.3 \%)$ are related to pollen exine development, nine $(52.9 \%)$ to pollen wall development, and two $(11.8 \%)$ to tapetum development. At the UNM stage, 11 (29.0\%) putatively up-regulated genes are related to pollen exine development and $27(71.0 \%)$ are related to other components of, or contributing to, pollen wall development, but none to tapetum development. In addition, there is one stage-enriched gene (Unigene6816) related to pollen exine development and one (Unigene9576) contributing to hemicellulose metabolic process, but none contributing to tapetum development. At the BCP stage, $50(21.4 \%)$ putatively up-regulated genes are related to pollen exine development, $180(76.9 \%)$ to other components of or contributing to pollen wall development and four (1.7 $\%)$ to tapetum development. At BCP stage, 20 (24.1\%) stageenriched genes are related to pollen exine development, 63 (75.9\%) to other components of or contributing to pollen wall development and none related to tapetum development. From TET stage onward, the genes related to pollen wall development first decrease in UNM then increase in BCP, while the genes related to tapetum development decrease in both UNM and BCP.
This trend is consistent with developmental events in pollen wall and tapetum.

\section{Identification of Putatively Stage-Specific Expressed Genes and Their Expression Profiles}

Pairwise comparisons within the four DGE libraries identified 7,720 genes are differentially expressed across the four stages. These genes were categorized into 14 groups based on up or down regulation. Hierarchical cluster analysis was performed on each of the groups to illustrate the results (Figure 7).

In group one to four, the genes are at least twofold upregulated in one particular stage comparing to any of the other three stages and the differences in expression level are not significant. In total, 1,644 (21.3\%), 1,637 (21.2\%), 688 $(8.9 \%)$, and $3,220(41.7 \%)$ genes illustrated in the groups one to four show expression peaks in MMC, TET, UNM, and $\mathrm{BCP}$, respectively. GO annotation analysis of the differentially expressed genes (Supplementary Table S6) indicated that a large number of genes were involved in oxidation-reduction processes, protein phosphorylation, pollen tube growth, response to salt stress, and response to cadmium ion. A total number of 86 genes $(1.1 \%)$ in the groups 5 and 10 show twofold upregulation in the former or the latter two stages, coinciding with the time of tapetum formation or tapetum PCD. These genes participate in oxidation-reduction processes, metabolic processes and DNA-dependent regulation of transcription. Genes in group 7 are down-regulated at tetrad and UNMs, while genes in group 8 are twofold up-regulated at these two stages. The genes in both groups were mainly enriched for protein ubiquitination. Genes in group 6 are down-regulated at tetrad and BCP stages, whereas group 9 genes are twofold up-regulated at these stages. Group 9 genes are involved in metabolic processes, sodium ion transmembrane transport, regulation of $\mathrm{pH}$, pollen tube growth, and plant-type cell wall modification. In groups 11 to 14, a total of 427 (5.5\%) genes are twofold down-regulated in one particular stage. The genes in these four groups were mainly related to oxidation-reduction process, ATP catabolic process, translation, DNA-dependent regulation of transcription. In total 3,427 (44.4\%) genes in all 14 groups were not annotated by GO terms and as such, deserve further attention as a source of genes with unidentified roles in anthers.

Groups one to four were further analyzed to identify stagespecifically enriched genes. They were defined as twofold upregulated and highly expressed $(\mathrm{RPKM} \geq 10)$ in one particular stage, while expression values in the other three stages were less than 10. As a result, 3,769 stage-specifically enriched genes across the four stages were obtained. The numbers at each stage were, $776(20.6 \%), 807(21.4 \%), 322(8.5 \%)$, and 1,864 $(49.5 \%)$ at MMC, TET, UNM, and BCP, respectively (Figure 8). UNM had the lowest share of stage-specifically enriched genes, while BCP had the largest share. Thus, BCP showed the most diverse expression profiles compared to the other three stages.

The expression patterns of all stage SEGs were analyzed and genes with similar expression profiles were clustered. 


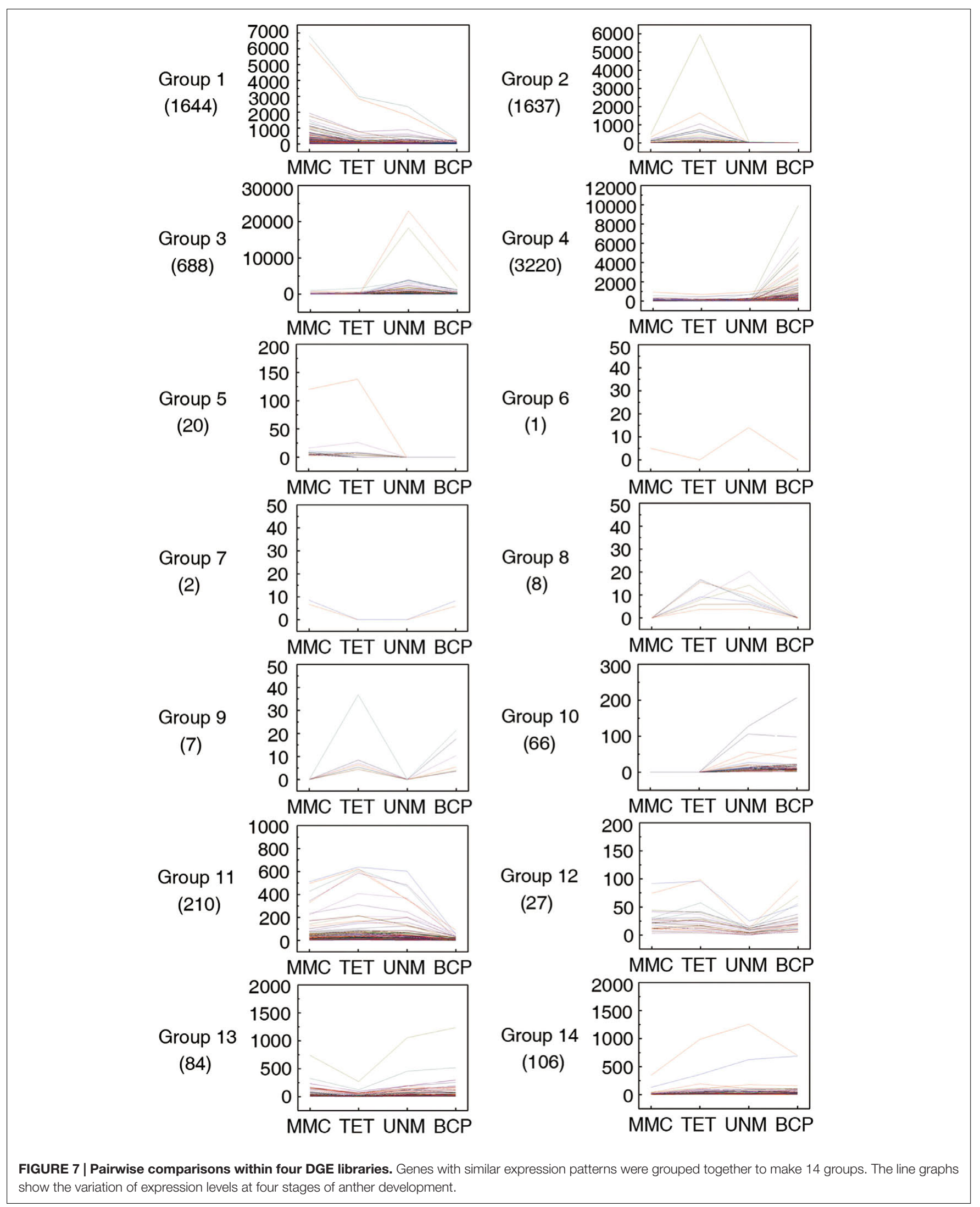




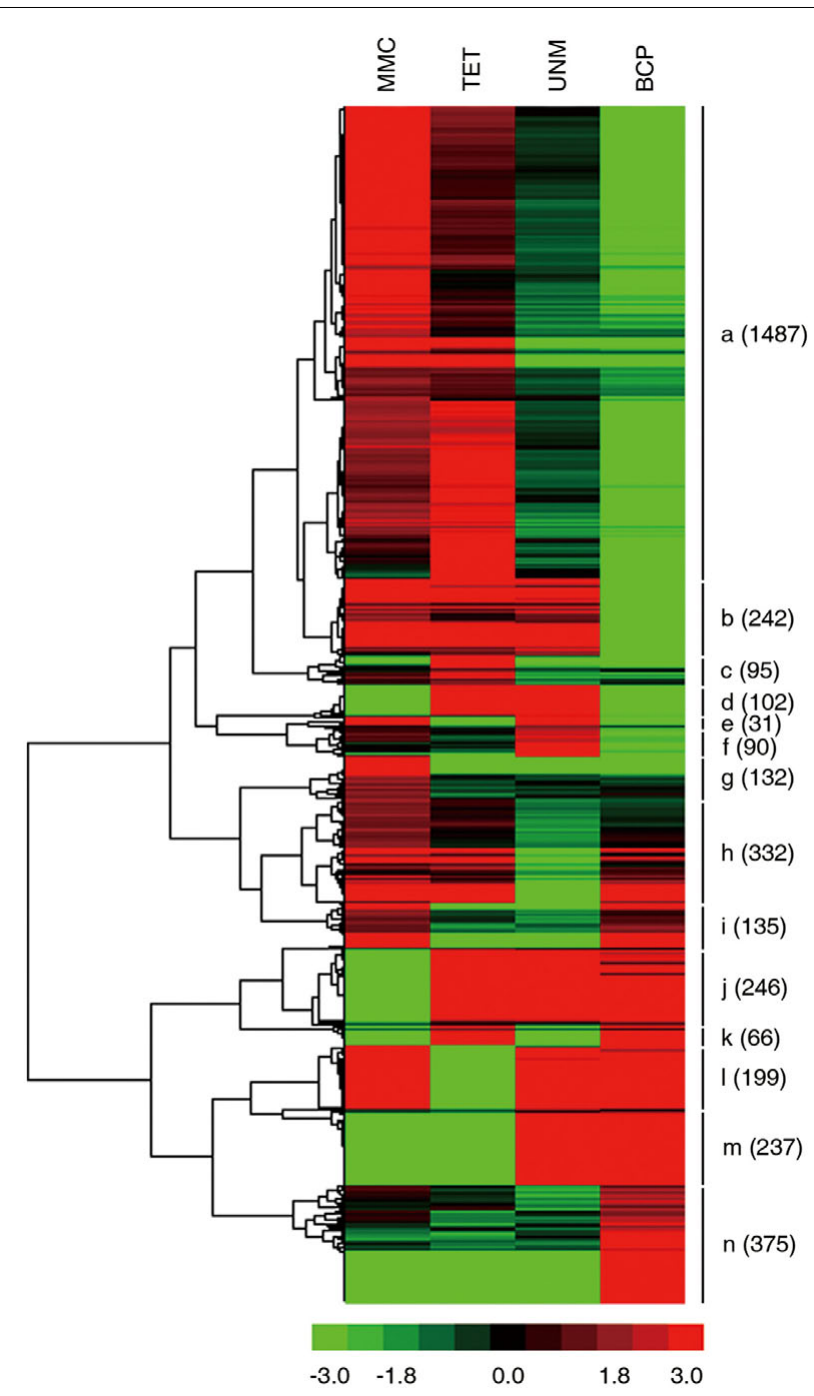

FIGURE 8 | Expression patterns of stage-specifically expressed genes. Hierarchical cluster diagram represents expression patterns of stage-specifically expressed genes of four stages. The numbers of genes under each cluster are showed at the right side.

A total of 14 clusters were generated (Figure 9). Genes in the same cluster are considered co-expressed and may be targets of the same TFs. The expression profile of the largest cluster ("a"; 1,487 genes) showed up-regulation in MMC and TET followed by down-regulation in UNM and BCP, coinciding with the pattern of tapetum development. Further, GO terms relating to tapetum development are strongly enriched in cluster "a" (Supplementary Table S7) confirming the cytological changes of tapetum development from MMC to BCP involve gene down-regulation. Clusters "b" (242) and "g" (132) also exhibit down-regulation from MMC to BCP and are dominated by genes related to oxidation-reduction process, DNA-dependent regulation of transcription and metabolic process. Clusters “j” (246), “m” (237), and “n” (375), show up-regulation trends from MMC to BCP and are dominated by genes related to oxidation-reduction process, pollen tube growth, plant-type cell wall modification, and cellular membrane fusion. In clusters " $d$ " (102), " $c$ " (95), and "f" (90), genes were up-regulated at both or either of the TET and UNM stages and annotated by lipid metabolic process, very longchain fatty acid metabolic process, transmembrane transport, and intracellular signal transduction. These features coincide with the finding that tapetal cells are most active during TET and UNM stages and are known to be involved in the synthesis of flavonoids and other secondary metabolites that eventually are transported to developing microspores. In clusters “i” (135), "l" (199), and "h" (332), genes are down-regulated at both or either of the TET and UNM stages and annotated by pollen tube growth, oxidationreduction process, plant-type cell wall modification, and negative regulation of PCD. Genes in cluster " $k$ " (66), showing upregulation at TET and BCP stages, are mainly involved in xylan biosynthetic process, glucuronoxylan metabolic process, plant-type cell wall modification, cellulose biosynthetic process, and plant-type cell wall biogenesis. Coincidently, callose wall, primexine, and intine related to polysaccharide metabolism are synthesized mainly at TET and BCP stages. Genes downregulated at TET and BCP stages are clustered in " $\mathrm{e}$ " (31), and are enriched in ATP catabolic process, metabolic process, transmembrane transport, and DNA-dependent regulation of transcription.

\section{Candidate Genes Relevant to Tapetum and Pollen Wall Development}

Differentially expressed genes and SEGs enriched for the relevant GO terms of anther wall and pollen wall development were identified. In total 243 differentially expressed genes and 108 stage-specific genes were obtained (Supplementary Figure S5). Genes related to sporopollenin biosynthetic processes are only enriched at TET (6) and UNM (1) stages. Genes related to pollen exine formation, are mainly enriched at TET (26 genes) and BCP (35 genes) stages, among which 5 (19.2\%) and 19 $(54.3 \%)$ genes are specifically expressed. Only one gene related to tapetal layer morphogenesis is specifically expressed at TET stage. There are four and three genes related to tapetal layer development differentially expressed at MMC and TET stages, respectively. Among those genes, three are specifically expressed at MMC and one is specifically expressed at TET stage. Genes relating to pectin are mainly enriched at $\mathrm{MMC}$ and $\mathrm{BCP}$ stages, with 14 (MMC) and 26 (BCP) genes being differentially expressed and 11 of which are specifically expressed at BCP stage. Genes relating to cellulose mainly express at MMC, TET, and BCP stages, with the number of differentially expressed genes being 15, 12, and 41, and the number of SEGs as 5, 4 , and 20, respectively. Genes corresponding to hemicellulose metabolic processes are only expressed at TET, UNM, and BCP stages, whilst $88.4 \%$ (38 genes) of these are differentially expressed at BCP stage and among these genes, 30 are specifically expressed. These findings are consistent with the cytological changes and developmental events in anther wall and pollen wall development. 


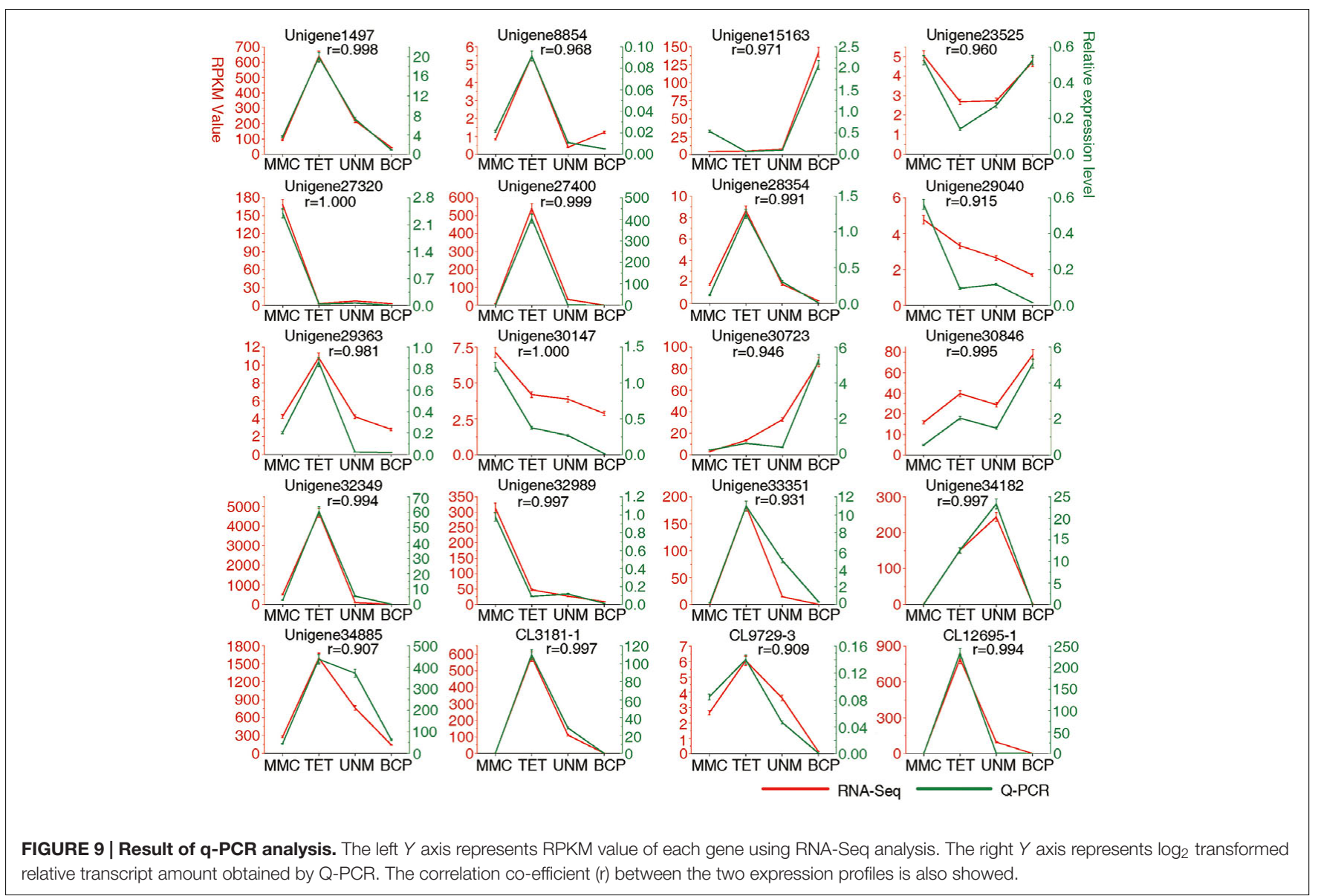

Pollen wall development requires lipid and polysaccharide metabolism, therefore genes enriched in carbohydrate and lipid metabolism pathways were analyzed (Supplementary Figure S6). The numbers of differentially expressed genes in both pathways only slightly change between MMC and UNM stages, while a marked increase occurs at BCP stage. This trend emphasizes the distinct gene expression profile at BCP stage. At MMC and UNM stages, a greater number of stage-enriched genes are involved in lipid metabolism in comparison to those involved in carbohydrate pathways (Supplementary Table S8). At TET and BCP stages, this trend is reversed, where fewer stage-enriched genes are involved in lipid metabolism than in carbohydrate metabolism. The MMC stage is enriched with a greater number of genes relating to glycerophospholipid and lipid metabolism, while TET stage is enriched in starch and sucrose metabolism as well as glycerophospholipid metabolism. UNM is enriched with the greatest number of genes relating to cutin, suberin, and wax biosynthesis. The first three largest numbers of genes at BCP are related to starch and sucrose metabolism, pentose and glucuronate interconversions, and glycerophospholipid metabolism. This might suggest nutrient synthesis and storage at $\mathrm{BCP}$ stage, consistent with the observation that the $\mathrm{BCP}$ cytoplasm is characterized by an abundance of starch grains and LD.

\section{Verification of Expression Profiles of Selected Unigenes Related to Tapetum and Pollen Wall Development by qRT-PCR}

Real-time quantitative PCR (qRT-PCR) analysis was carried out on 20 candidate genes selected at random (Figure 9). Among these, 14 genes show high expression with RPKM $\geq 10$ in at least one stage, while six display low expression with RPKM $\leq 10$ at all four stages. The expression values at developmental stages derived from RNA-seq and q-PCR of each gene are listed in Supplementary Table S9. The expression patterns of all 20 genes analyzed by qRT-PCR largely agree with the DGE tag profiling, as the correlation co-efficients $(r)$ are all greater than 0.9. This result provides support for the reliability of RNA-Seq data and the differential gene expression profiles observed for anther stages of H. patens.

\section{DISCUSSION}

\section{Tapetum and Pollen Development Display Common Features in Rubiaceae}

Anther development is a complex process which involves transition from sporophyte to gametophyte, control of mitotic 
and meiotic cell divisions, together with the coordination of pollen and anther maturation (McCormick, 1993; Scott et al., 2004; Zhang et al., 2011). Ultrastructural changes involved in anther development have been described for many angiosperms, especially for model species including Arabidopsis and rice (Sanders et al., 1999; Zhang and Wilson, 2009; Zhang et al., 2011), although there are differences in the resolution and detail of the stages described (Owen and Makaroff, 1995). In our study, anther and pollen development of $H$. patens was shown to be typical of many angiosperms, with some features common in the Rubiaceae family. Our observations of developmental events and cytological changes provide a solid foundation for the analysis of gene expression profiles in the developing anthers of $H$. patens.

The tapetum cells in $H$. patens maintain integrity and position indicating that the tapetum is of the secretory type, which fits the description of a type five tapetum (Pacini, 1997), the most common in angiosperms (Owen and Makaroff, 1995). Ubisch bodies occurred simultaneously with the developing pollen exine, which corroborates the idea that they are required for transferring tapetum-derived sporopollenin precursors to the exine (Huysmans et al., 1998). The tapeta of Arabidopsis and rice are also secretory but use different export routes for sporopollenin. In Arabidopsis, specialized organelles, elaioplasts, and tapetosomes, are supposed to be transporters. Lipids are transported from the tapetum to microspores in two ways: vesicular transport and use of transporters as lipid carriers (Vizcay-Barrena and Wilson, 2006). In rice and other cereals, Ubisch bodies are thought to export sporopollenin across the hydrophilic cell wall to the locule (Zhang et al., 2011). Thus, the tapetum in dicots and monocots might have different mechanisms for sporopollenin translocation. Analogies are often found between the ornamentation of the pollen exine and that of the Ubisch body wall (Hesse, 1986; Vinckier and Smets, 2002, 2003). Ubisch bodies are considered have great potential as model system to study sporopollenin deposition, since they are acellular structures, independent of cytoplasmic control (Clément and Audran, 1993; Verstraete et al., 2014). To date, only the RAFTIN gene was identified in pro-orbicule bodies and shown to accumulate in Ubisch bodies in wheat and rice. RAFTIN is highly anther-specific and essential for pollen development in cereals (Wang et al., 2003). Future genetic studies to identify the genes and gene products that control sporopollenin polymerisation and exine patterning will need to take Ubisch bodies into account when screening for phenotypes (Verstraete et al., 2014).

The fundamental structure of pollen wall in angiosperms consisting of the outer exine and the inner intine is generally conserved (Heslop-Harrison, 1968; Blackmore et al., 2007; Ariizumi and Toriyama, 2011). The components contributing to the pollen wall are produced and accumulated in a precise temporal sequence. Sporopollenin makes up the majority of the material of the exine (Blackmore et al., 2007). In H. patens, the sporopollenin deposition starts from TET stage. Intine development starts from the late UNM stage and is complete by the end of BCP stage. The ontogenetic sequence of pollen wall development follows the basic scheme in the family (Hansson and El-Ghazaly, 2000; El-Ghazaly et al., 2001). Intinous projections occurred in the aperture region of $H$. patens $P G$, known as protruding onci or pollen buds in the Rubiaceae, was believed to be a relatively common feature in this family (Dessein et al., 2005; Kuang et al., 2012).

Besides exine and intine, the callose wall and primexine are successively synthesized and degraded at precise times. The callose wall, consisting of linear $\beta$-1,3-glucan polymers (Hird et al., 1993), begins to deposit at the beginning of TET stage and degrades at the end of TET stage in H.patens. The primexine, which acts as a template that guides the accumulation of sporopollenin, is composed of neutral and acidic polysaccharides, cellulose, and some proteins (Heslop-Harrison, 1968; Scott et al., 2004; Jiang et al., 2013). Primexine matrix usually with fibrillary texture appears between the callose and the plasmalemma at the TET stage in $H$. patens. In addition, lipidic material, which may be classed as pollenkitt (El-Ghazaly et al., 2001; Edlund et al., 2004), is deposited onto the exine surface at the late BCP stage. These ontogenetical features were also described in the available ultrastructural studies in Rubiaceae (Hansson and El-Ghazaly, 2000; El-Ghazaly et al., 2001; Kuang and Liao, 2015).

\section{DEGs Expression Patterns Agree with the Developmental Events}

Genes related to sporopollenin biosynthetic process were closely associated with fatty acid metabolism and only expressed at TET and UNM stages, consistent with the appearance of proUbisch bodies and Ubisch bodies in tapetal cells. Genes related to pollen exine formation are enriched at TET and BCP stages, which may correspond to the time that primexine formed and exine deposited. Genes related to hemicellulose metabolic process, are mainly enriched at BCP stage, a few are enriched at TET and UNM stages and none are expressed at MMC stage, coinciding with the stages at which the primary cell wall and intine develop. Genes related to pectin are largely enriched at BCP stage, and genes related to cellulose are largely enriched at TET and BCP stages. These gene expression patterns agree well with the observed stages in which intine and primexine appear in $H$. patens. Genes related to tapetal layer morphogenesis and development are enriched at MMC and TET stages, consistent with stages during which tapetal cells are differentiated and pro-Ubisch bodies are produced.

The numbers of genes enriched in different pathways are correlated with structures and components present at different anther developmental stages. At UNM stage, abundant Ubisch bodies are synthesized, which involve lipid metabolism. Consistently, more genes are revealed in lipid metabolism pathways than in carbohydrate metabolism pathways at the same stage. The synthesis and degradation of callose wall and primexine, and the formation of intine are involved in carbohydrate metabolic pathways (Jiang et al., 2013). The genes related to the carbohydrate metabolism are enriched at the stages during which callose wall, primexine, and intine form. In addition, the cytoplasm of mature $H$. patens PG is characterized by an abundance of compound starch grains and $\mathrm{LD}$ at $\mathrm{BCP}$ stage. This may account for the increase in the number of genes enriched in both carbohydrate and lipid metabolism pathways at this stage. 
Gene ontology enrichment analysis of the stage-enriched genes and differentially expressed genes suggested the gene number, gene function, and expression profile were significantly correlated with anther developmental processes in $H$. patens. The genes filtered from KEGG pathways analysis and GO enrichment analysis, provide a useful resource to identify novel genes and their possible functions in pollen wall development.

\section{Comparative Analysis of Anther and Pollen Transcriptomes Confirms Inter-Specific Conserved Patterns}

Developmental transcriptomic analysis of anthers and PG utilizing microarray platforms have been reported in Arabidopsis (Honys and Twell, 2004; Pina et al., 2005), rice (Huang et al., 2009; Fujita et al., 2010; Deveshwar et al., 2011), maize (Ma et al., 2008), and Brassica rapa (Dong et al., 2013). Our study revealed 89,849 unigenes in the $H$. patens anther transcriptome, well above the numbers of genes predicted to be expressed in anthers of other species. However, high-throughput sequencing techniques increase the chance of detecting transcripts with low abundance and microarrays have been reported to be less effective at identifying low abundance transcripts (Loraine et al., 2013). Further, the number of $H$. patens unigenes is an estimate, since the genome is unknown and unigenes sharing sequence similarities were classified as different unigenes, in consideration of alternative splicing events. High numbers of unigenes have also been reported for species with no reference genomes such as for the fruit transcriptome of Triadica sebifera with 92,550 unigenes (Divi et al., 2016), the embryo transcriptome of chrysanthemum with 116,697 unigenes (Zhang F.J. et al., 2014) and the leaf transcriptome of Lycium chinense with 61,595 unigenes (Wang et al., 2015). Finally, each developmental stage of $H$. patens anther contains about $20 \%$ of transcripts with RPKM $\leq 1$, which most likely would not be detected with microarrays. These low abundance transcripts provide a resource for future analyses.

Transcriptomic analysis in developing and germinated pollen of rice displayed a "U-type" change in the number with the lowest number at the BCP stage (Wei et al., 2010). Developmental analysis of the Arabidopsis pollen transcriptome revealed a major shift in mRNA populations between BCP and TCP stages, reflective of the transition from earlier cell division to later pollen maturity (Honys and Twell, 2004). This distinct phase shift suggests inter-specific conservation of pollen-expressed genes. In $H$. patens anthers, BCP stage has the greatest number of stage-specific genes. The divergence between $H$. patens and the two model species might result from the relative contribution of sporophytic and gametophytic cell types and earlier developmental stages (MMC and TET) in H. patens together with taxon-specific features, such as insect-pollination and BCP at maturity. Moreover, different platforms, tissue collections and comparison methods can result in significant variation in anther transcriptome profiles for different species (Hollender et al., 2014).

Since sample staging of anther development is similar between $H$. patens and rice in the study of Deveshwar et al. (2011), additional comparative analysis was carried out. Transcriptome profiling of rice was investigated in anthers at pre-meiotic (PMA), meiotic (MA), single-celled microspore (SCP), and tri-nucleate pollen (TPA) stages (Deveshwar et al., 2011). At PMA and MA stages (MMC in $H$. patens), there was high representation of sporophytic tissue compared to gametophytic tissue. This characteristic coincided with the phenomenon that most of the transcriptome changes corresponding to the sporogenous tissue and developing tapetum. TPA anthers contained a relatively higher cellular mass of gametophytic tissue (pollen), meanwhile prominent differences were found in transcript composition comparing with transcriptomes of earlier anther stages. In $H$. patens, BCP stage anthers were the most distinctive of the four stages, with the highest number of stage-specific and differentially expressed transcripts (Figure 10), showing great similarity to the TPA stage in rice (Deveshwar et al., 2011). This common shift, from free microspore stage anthers to late stage anthers, could be explained by the distinctive transcriptomes of developing pollen and sperm. It also reflects the large number of BCP enriched genes in $H$. patens associated with pollen development and maturation. Comparison of gene expression between two adjacent stages of anther development further highlighted this major switch of gene expression. The apparent transition from the free microspore stage to the BCP stage in $H$. patens also reflected the switch from the sporophytic to the gametophytic program (Deveshwar et al., 2011).

\section{Homologs of Anther and Pollen-Expressed Transcription Factors Were Revealed}

Putative homologs of known TFs expressed in developing anthers were identified in $H$. patens (Supplementary Table S10). The expression patterns of these genes were largely consistent with those of their counterparts in other species, supporting the conservation of regulatory features in anther and pollen development in $H$. patens.

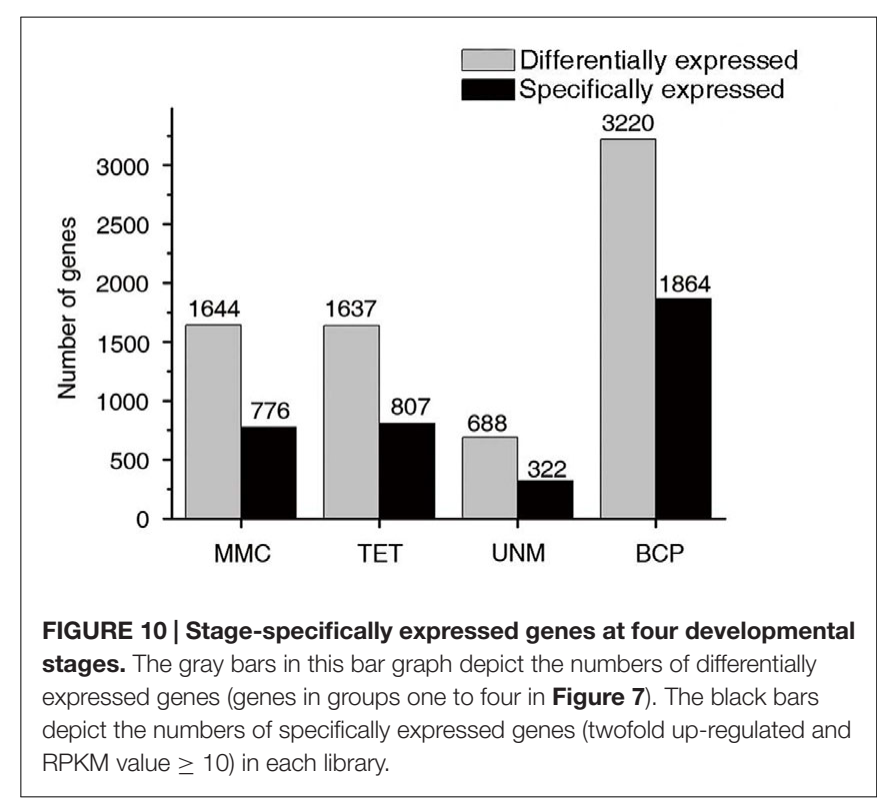


In the early stages of Arabidopsis anther development, DYT1 is required for tapetum development and function (Zhang et al., 2006), directly regulating TDF1 expression in the tapetum (Feng et al., 2012). The highest expression of both DYT1 and TDF1 occurred at similar stages of tapetal development (Gu et al., 2014). CL1502.contig1 of Hamelia patens shares high amino acid sequence identity with DYT1 in the bHLH domain. Unigene29472 is a putative homolog of TDF1. Both CL1502.contig1 and Unigene29472 show expression peaks at MMC stage, weak expression at TET or/and UNM stages, but no expression at BCP stage. AtMYB103, another important TF for tapetum function and male fertility, is also regulated by DYT1 (Feng et al., 2012). A candidate homolog of AtMYB103, CL6214.contig1, shows peak expression at MMC stage and lower expression at TET stage, but no expression at UNM and BCP stages. The expression pattern of these three putative homologs correspond to the early stages of tapetum development and callose dissolution, and exhibit somewhat consistent patterns with those of DYT1, AtMYB103, and TDF1, which suggests shared pathways in the regulatory network controlled by DYT1 in Arabidopsis and $H$. patens.

CL4992.contig3 shares high amino acid sequence identity with the MYB domains of AtMYB97, AtMYB101, and AtMYB120. It shows expression peak at BCP stage, indicating a consistent profile with these three MYB genes. DUO POLLEN1 (DUO1) is an Arabidopsis male germline-specific R2R3-type MYB TF (Durbarry et al., 2005; Rotman et al., 2005) that is essential for male germ cell division and differentiation (Brownfield et al., 2009). CL1108.Contig1, homologous to AtDUO1, shows peak expression at BCP stage, consistent with that of AtDUO1. It is likely that some of the pathways regulated by MYB factors are shared by Arabidopsis and H. patens.

The AtMIKC* TFs AGAMOUS-LIKE30 (AGL30), AGL65, AGL66, AGL94, and AGL104 are mainly expressed in mature pollen and play an essential role in transcriptional regulation during late pollen development (Verelst et al., 2007a,b). In $H$. patens, Unigene32598 and Unigene1629 share high amino acid sequence identity in the MIKC* domain, representing probable homologs of MIKC* TFs. Both of these are predominantly expressed at BCP stage, consistent with the expression patterns of AtMIKC* genes, which indicates conserved regulatory features of late pollen development and pollen maturation in H. patens.

\section{Expression Profiles of Homologs with Verified Conserved Patterns in Pollen Development}

The $H$. patens homologs of known genes with characterized functions in exine formation in Arabidopsis or rice were identified (Supplementary Table S10). Their expression profiles were analyzed and verified by Q-PCR. TKPR1, which is involved in a conserved biosynthetic pathway in sporopollenin monomer biosynthesis, shows peak expression at tetrad stage (Grienenberger et al., 2010). Unigene32349, a homolog of TKPR1, is putatively highly expressed at TET stage, consistent with the expression profile of TKPR1. LAP6 and LAP5 are specifically expressed during the period of exine synthesis and are essential for the exine production (Dobritsa et al., 2010). The homologous unigenes CL12695Contig1 and CL3181Contig1 show peak expression at TET and UNM stages. LAP3 is essential for pollen development and proper exine formation (Dobritsa et al., 2009), and the homologous Unigene 27400 shows peak expression at TET stage. Unigene 34182 shows peak expression at TET and UNM stages and represents the $H$. patens homolog of MS2, which is involved in exine development and is expressed in tapetum shortly after microspore release (Aarts et al., 1997; Gómez et al., 2015). Unigene33351 and CL9729-Contig3 show higher read counts at TET stage and are homologous to the gene $A B C G 26 / W B C 27$. ABCG26 is expressed specifically in tapetal cells at the early vacuolate stage and plays a crucial role in the transfer of sporopollenin lipid precursors from tapetal cells to anther locules (Choi et al., 2011), while WBC27 is expressed during early stages of anther development (Xu et al., 2010).

Unigenes homologous to known genes related to tapetum development, intine development and callose synthesis were also identified in $H$. patens (Supplementary Table S10). Unigene30846, homologous to AtUSP (Schnurr et al., 2006), is suggested to be involved in intine development and putatively highly expressed at BCP stage, consistent with the period of intine accumulation. Moreover, Unigene30846 matches Cc10_g13640 of coffee and shares the functional GO annotation of UDP-sugar pyrophosphorylase, which suggests functional similarity. Unigene29363 is homologous to CalS5, which encodes a callose synthase (Dong et al., 2005). The annotation of Unigene29363 (0052543//callose deposition in cell wall, $0006075 / /(1->3)$-beta-D-glucan biosynthetic process and 0009556//microsporogenesis) and peak expression at TET stage are consistent with the function and expression pattern of CalS5. RTS2, a unique gene in the rice genome, is required for tapetal development and is predominantly expressed during meiosis (Luo et al., 2006). Unigene8854 is homologous to RTS2 and shows peak expression at TET stage corresponding to that of RTS2.

Collectively, the predicted functions and verified expression patterns of the aforementioned unigenes are consistent with those of their homologs in Arabidopsis and/or in rice. This phylogenetic conservation of gene expression further validates our analysis of the $H$. patens anther transcriptome.

\section{Potential Genes for Tapetum and Pollen Wall Development Provide Useful Resources for Future Study}

Currently most characterized genes involved in tapetum or pollen wall development were identified based on genetic analysis of male sterile or reduced fertility mutants, including those with defective pollen wall development. Transcriptomic profiling is expected to expand knowledge of the genes involved in these developmental processes. In particular, our analysis of the $H$. patens anther transcriptome has allowed the identification of many more candidate genes involved in 
pollen development. We identified 243 differentially expressed genes and 108 stage-specific genes potentially related to tapetum layer morphogenesis and development, sporopollenin biosynthesis, exine formation, cellulose and pectin metabolism and biosynthesis, and hemicellulose and cellulose metabolism. The most significant alignments among these classes of genes derive from Solanum lycopersicon, Vitis vinifera, and Coffea canephora. Moreover, some potential orthologs in C. canephora have similar annotations relevant to pollen development, for instance, callose synthase and cellulose synthase. Low abundance transcripts expressed during anther development were also mined and differential expression patterns of genes in anthers and pollen were uncovered. This wealth of information lays the foundation for higher resolution genome-wide transcriptomic profiling of $H$. patens, functional investigation of the identified candidate genes, and the evo-devo exploration of angiosperm pollen.

\section{DATA ACCESSIBILITY}

Clean Illumina reads: NCBI SRA: SRP079620.

\section{AUTHOR CONTRIBUTIONS}

YK conceptualized and designed this study. LY and YK performed the experiments. LY, YK, XZ, and DT performed data analysis. LY, YK, DT, and JL wrote the manuscript. All authors have read and approved the final version of the manuscript.

\section{FUNDING}

This work was supported by National Natural Science Foundation of China (31200176, 31470410, 31670336). Guangdong Provincial Key Laboratory of Applied Botany, the

\section{REFERENCES}

Aarts, M. G., Hodge, R., Kalantidis, K., Florack, D., Wilson, Z. A., Mulligan, B. J., et al. (1997). The Arabidopsis MALE STERILITY 2 protein shares similarity with reductases in elongation/condensation complexes. Plant J. 12, 615-623. doi: 10.1046/j.1365-313X.1997.00615.x

APG III (2009). An update of the Angiosperm Phylogeny Group classification for the orders and families of flowering plants: APG III. Bot. J. Linn. Soc. 161, 105-121. doi: 10.1111/j.1095-8339.2009. 00996.x

Ariizumi, T., and Toriyama, K. (2011). Genetic regulation of sporopollenin synthesis and pollen exine development. Annu. Rev. Plant Biol. 62, 437-460. doi: 10.1146/annurev-arplant-042809-112312

Audic, S., and Claverie, J. M. (1997). The significance of digital gene expression profiles. Genome Res. 7, 986-995. doi: 10.1101/gr.7.10.986

Aya, K., Ueguchi-Tanaka, M., Kondo, M., Hamada, K., Yano, K., Nishimura, M., et al. (2009). Gibberellin modulates anther development in rice via the transcriptional regulation of GAMYB. Plant Cell 21, 1453-1472. doi: 10.1105/ tpc.108.062935

Barrero, R. A., Chapman, B., Yang, Y., Moolhuijzen, P., Keeble-Gagnere, G., Zhang, N., et al. (2011). De novo assembly of Euphorbia fischeriana root
National Science and Technology Infrastructure Program of China-Compilation of Ex Situ Cultivated Flora of Botanical Garden (2015FY210100), Guangdong Provincial Key Laboratory of Digital Botanical Garden and Key Laboratory of Plant Resources Conservation and Sustainable Utilization, SCBG, CAS.

\section{ACKNOWLEDGMENT}

We thank Ms. Rufang Deng and Ms. Xiaoying $\mathrm{Hu}$ in the SCBG for technical assistances in TEM and SEM analysis, respectively, and the two reviewers for their helpful comments.

\section{SUPPLEMENTARY MATERIAL}

The Supplementary Material for this article can be found online at: http://journal.frontiersin.org/article/10.3389/fpls.2016.01991/ full\#supplementary-material

TABLE S1 | Primers used in q-PCR.

TABLE S2 | Result of KEGG annotation.

TABLE S3 | Alignment result of the $\boldsymbol{H}$. patens unigenes with the coffee genome.

TABLE S4 | GO annotation of up-regulated and stage-enriched genes.

TABLE S5 | GO terms selected for GO functional enrichment analysis.

TABLE S6 | GO annotation of differentially expressed genes.

TABLE S7 | GO annotation of clusters a-n.

TABLE S8 | Numbers of genes enriched in carbohydrate and lipid metabolism pathways.

TABLE S9 | Expression values from RNA-seq and q-PCR of each gene.

TABLE S10 | Putative homologs of known TFs expressed in $\boldsymbol{H}$. patens anthers.

transcriptome identifies prostratin pathway related genes. BMC Genomics 12: 600. doi: 10.1186/1471-2164-12-600

Blackmore, S., Wortley, A. H., Skvarla, J. J., and Rowley, J. R. (2007). Pollen wall development in flowering plants. New Phytol. 174, 483-498. doi: 10.1111/j. 1469-8137.2007.02060.x

Blanca, J., Canizares, J., Roig, C., Ziarsolo, P., Nuez, F., and Picó, B. (2011). Transcriptome characterization and high throughput SSRs and SNPs discovery in Cucurbita pepo (Cucurbitaceae). BMC Genomics 12: 104. doi: 10.1186/14712164-12-104

Boavida, L. C., Becker, J. D., and Feijo, J. A. (2005). The making of gametes in higher plants. Int. J. Dev. Biol. 49, 595-614. doi: 10.1387/ijdb. 052019lb

Bokvaj, P., Hafidh, S., and Honys, D. (2015). Transcriptome profiling of male gametophyte development in Nicotiana tabacum. Genomic Data 3, 106-111. doi: $10.1016 /$ j.gdata.2014.12.002

Borg, M., and Twell, D. (2011). "Pollen: structure and development," in eLS (Encyclopedia of Life Sciences), ed. A. F. Agró (Chichester: John Wiley \& Sons, Ltd.). doi: 10.1002/9780470015902.a0002039.pub2

Brownfield, L., Hafidh, S., Borg, M., Sidorova, A., Mori, T., and Twell, D. (2009). A plant germline-specific integrator of sperm specification and cell cycle progression. PLoS Genet 5:e1000430. doi: 10.1371/journal.pgen.1000430 
Choi, H., Jin, J. Y., Choi, S., Hwang, J. U., Kim, Y. Y., Suh, M. C., et al. (2011). An ABCG/WBC-type ABC transporter is essential for transport of sporopollenin precursors for exine formation in developing pollen. Plant J. 65, 181-193. doi: 10.1111/j.1365-313X.2010.04412.x

Clément, C., and Audran, J. C. (1993). Orbicule wall surface characteristics in Lilium (Liliaceae). An ultrastructural and cytochemical approach. Grana 32, 348-353. doi: 10.1080/00173139309428963

Conesa, A., Gotz, S., Garcia-Gomez, J. M., Terol, J., Talon, M., and Robles, M. (2005). Blast2GO: a universal tool for annotation, visualization and analysis in functional genomics research. Bioinformatics 21, 3674-3676. doi: 10.1093/ bioinformatics/bti610

De Hoon, M. J. L., Imoto, S., Nolan, J., and Miyano, S. (2004). Open source clustering software. Bioinformatics 20, 1453-1454. doi: 10.1093/bioinformatics/ bth078

Denoeud, F., Carretero-Paulet, L., Dereeper, A., Droc, G., Guyot, R., Pietrella, M., et al. (2014). The coffee genome provides insight into the convergent evolution of caffeine biosynthesis. Science 345, 1181-1184. doi: 10.1126/science. 1255274

Dereeper, A., Bocs, S., Rouard, M., Guignon, V., Ravel, S., Tranchant-Dubreuil, C., et al. (2015). The coffee genome hub: a resource for coffee genomes. Nucleic Acids Res. 43, D1028-D1035. doi: 10.1093/nar/gku1108

Dessein, S., Ochoterena, H., De Block, P., Lens, F., Robbrecht, E., Schols, P., et al. (2005). Palynological characters and their phylogenetic signal in Rubiaceae. Bot. Rev. 73, 354-414. doi: 10.1663/0006-8101(2005)071[0354:PCATPS]2. $0 . \mathrm{CO} ; 2$

Deveshwar, P., Bovill, W. D., Sharma, R., Able, J. A., and Kapoor, S. (2011). Analysis of anther transcriptomes to identify genes contributing to meiosis and male gametophyte development in rice. BMC Plant Biol. 11: 78. doi: 10.1186/14712229-11-78

Divi, U. K., Zhou, X. R., Wang, P., Butlin, J., Zhang, D. M., Liu, Q., et al. (2016). Deep sequencing of the fruit transcriptome and lipid accumulation in a nonseed tissue of Chinese tallow, a potential biofuel crop. Plant Cell Physiol. 57, 125-137. doi: 10.1093/pcp/pcv181

Dobritsa, A. A., Lei, Z., Nishikawa, S. I., Urbanczyk-Wochniak, E., Huhman, D. V., Preuss, D., et al. (2010). LAP5 and LAP6 encode anther-specific proteins with similarity to chalcone synthase essential for pollen exine development in Arabidopsis thaliana. Plant Physiol. 153, 937-955. doi: 10.1104/pp.110.157446

Dobritsa, A. A., Nishikawa, S. I., Preuss, D., Urbanczyk-Wochniak, E., Sumner, L. W., Hammond, A., et al. (2009). LAP3, a novel plant protein required for pollen development, is essential for proper exine formation. Sex. Plant Reprod. 22, 167-177. doi: 10.1007/s00497-009-0101-8

Dong, X. S., Feng, H., Xu, M., Lee, J., Kim, Y. K., Lim, Y. P., et al. (2013). Comprehensive analysis of genic male sterility-related genes in Brassica rapa using a newly developed br300k oligomeric chip. PLOS ONE 8: 24. doi: 10.1371/ journal.pone.0072178

Dong, X. Y., Hong, Z. L., Sivaramakrishnan, M., Mahfouz, M., and Verma, D. P. S. (2005). Callose synthase (CalS5) is required for exine formation during microgametogenesis and for pollen viability in Arabidopsis. Plant J. 42, 315-328. doi: 10.1111/j.1365-313X.2005.02379.x

Dukowic-Schulze, S., and Chen, C. (2014). The meiotic transcriptome architecture of plants. Front. Plant Sci. 5: 220. doi: 10.3389/fpls.2014.00220

Durbarry, A., Vizir, I., and Twell, D. (2005). Male germ line development in Arabidopsis. Duo pollen mutants reveal gametophytic regulators of generative cell cycle progression. Plant Physiol. 137, 297-307. doi: 10.1104/pp.104.053165

Edlund, A. F., Swanson, R., and Preuss, D. (2004). Pollen and stigma structure and function: the role of diversity in pollination. Plant Cell 16, S84-S97. doi: $10.1105 /$ tpc. 015800

El-Ghazaly, G., Huysmans, S., and Smets, E. F. (2001). Pollen development of Rondeletia odorata (Rubiaceae). Am. J. Bot. 88, 14-30. doi: 10.2307/2657122

Feng, B. M., Lu, D. H., Ma, X., Peng, Y. B., Sun, Y. J., Ning, G., et al. (2012). Regulation of the Arabidopsis anther transcriptome by DYT1 for pollen development. Plant J. 72, 612-624. doi: 10.1111/j.1365-313X.2012.05104.X

Fujita, M., Horiuchi, Y., Ueda, Y., Mizuta, Y., Kubo, T., Yano, K., et al. (2010). Rice expression atlas in reproductive development. Plant and Cell Physiol. 51, 2060-2081. doi: 10.1093/pcp/pcq165

Garg, R., Patel, R. K., Tyagi, A. K., and Jain, M. (2011). De novo assembly of chickpea transcriptome using short reads for gene discovery and marker identification. DNA Res. 18, 53-63. doi: 10.1093/dnares/dsq028
Goldberg, R. B., Beals, T. P., and Sanders, P. M. (1993). Anther development - basic principles and practical applications. Plant Cell 5, 1217-1229. doi: 10.1105/tpc. 5.10.1217

Gómez, J. F., Talle, B., and Wilson, Z. A. (2015). Anther and pollen development: a conserved developmental pathway. J. Integr. Plant Biol. 57, 876-891. doi: $10.1111 /$ jipb. 12425

Grabherr, M. G., Haas, B. J., Yassour, M., Levin, J. Z., Thompson, D. A., Amit, I., et al. (2011). Full-length transcriptome assembly from RNA-Seq data without a reference genome. Nat. Biotechnol. 29, 644-652. doi: 10.1038/ nbt. 1883

Grienenberger, E., Kim, S. S., Lallemand, B., Geoffroy, P., Heintz, D., de Azevedo Souza, C., et al. (2010). Analysis of TETRAKETIDE $\alpha$-PYRONE REDUCTASE function in Arabidopsis thaliana reveals a previously unknown, but conserved, biochemical pathway in sporopollenin monomer biosynthesis. Plant Cell 22, 4067-4083. doi: 10.1105/tpc.110. 080036

Gu, J. N., Zhu, J., Yu, Y., Teng, X. D., Lou, Y., Xu, X. F., et al. (2014). DYT1 directly regulates the expression of TDF1 for tapetum development and pollen wall formation in Arabidopsis. Plant J. 80, 1005-1013. doi: 10.1111/tpj. 12694

Guo, Q. Q., Ma, X. J., Wei, S. G., Qiu, D. Y., Wilson, I. W., Wu, P., et al. (2014). De novo transcriptome sequencing and digital gene expression analysis predict biosynthetic pathway of rhynchophylline and isorhynchophylline from Uncaria rhynchophylla, a non-model plant with potent antialzheimer's properties. BMC Genomics 15: 676. doi: 10.1186/1471-216415-676

Hansson, T., and El-Ghazaly, G. (2000). Development and cytochemistry of pollen and tapetum in Mitriostigma axillare (Rubiaceae). Grana 39, 65-89. doi: 10. 1080/001731300300045184

Heslop-Harrison, J. (1968). Pollen wall development. Science 161, 230-237. doi: $10.1126 /$ science. 161.3838 .230

Hesse, M. (1986). Orbicules and the ektexine are homologous sporopollenin concretions in Spermatophyta. Plant Syst. Evol. 153, 37-48. doi: 10.1007/ BF00989416

Higginson, T., Li, S. F., and Parish, R. W. (2003). AtMYB103 regulates tapetum and trichome development in Arabidopsis thaliana. Plant J. 35, 177-192. doi: 10.1046/j.1365-313X.2003.01791.x

Hird, D. L., Worrall, D., Hodge, R., Smartt, S., Paul, W., and Scott, R. (1993). The anther-specific protein encoded by the Brassica napus and Arabidopsis thaliana a6-gene displays similarity to beta-1,3-glucanases. Plant J. 4, 1023-1033. doi: 10.1046/j.1365-313X.1993.04061023.x

Hollender, C. A., Kang, C., Darwish, O., Geretz, A., Matthews, B. F., Slovin, J., et al. (2014). Floral transcriptomes in woodland strawberry uncover developing receptacle and anther gene networks. Plant Physiol. 165, 1062-1075. doi: 10. 1104/pp.114.237529

Honys, D., and Twell, D. (2004). Transcriptome analysis of haploid male gametophyte development in Arabidopsis. Genome Biol. 5: R85. doi: 10.1186/ gb-2004-5-11-r85

Hu, J., Wang, Z. D., Zhang, L. Y., and Sun, M. X. (2014). The Arabidopsis Exine Formation Defect (EFD) gene is required for primexine patterning and is critical for pollen fertility. New Phytol. 203, 140-154. doi: 10.1111/nph. 12788

Hua, W. P., Zhang, Y., Song, J., Zhao, L. J., and Wang, Z. Z. (2011). De novo transcriptome sequencing in Salvia miltiorrhiza to identify genes involved in the biosynthesis of active ingredients. Genomics 98, 272-279. doi: 10.1016/j.ygeno. 2011.03.012

Huang, M. D., Hsing, Y. 1, and Huang, A. H. (2011). Transcriptomes of the anther sporophyte: availability and uses. Plant Cell Physiol. 52, 1459-1466. doi: $10.1093 / \mathrm{pcp} / \mathrm{pcr} 088$

Huang, M. D., Wei, F. J., Wu, C. C., Hsing, Y. I. C., and Huang, A. H. C. (2009). Analyses of advanced rice anther transcriptomes reveal global tapetum secretory functions and potential proteins for lipid exine formation. Plant Physiol. 149, 694-707. doi: 10.1104/pp.108.131128

Huysmans, S., El-Ghazaly, G., and Smets, E. (1998). Orbicules in angiosperms: morphology, function, distribution, and relation with tapetum types. Bot. Rev. 64, 240-272. doi: 10.1007/BF02856566

Iseli, C., Jongeneel, C. V., and Bucher, P. (1999). "ESTScan: a program for detecting, evaluating, and reconstructing potential coding regions in EST sequences," in 
Proceedings of the International Conference on Intelligent Systems for Molecular Biology, Heidelberg, 138-148.

Jiang, J., Zhang, Z., and Cao, J. (2013). Pollen wall development: the associated enzymes and metabolic pathways. Plant Biol. 15, 249-263. doi: 10.1111/j.14388677.2012.00706.x

Kanehisa, M., Araki, M., Goto, S., Hattori, M., Hirakawa, M., Itoh, M., et al. (2008). KEGG for linking genomes to life and the environment. Nucleic Acids Res. 36, D480-D484. doi: 10.1093/nar/gkm882

Kapoor, S., Kobayashi, A., and Takatsuji, H. (2002). Silencing of the tapetumspecific zinc finger gene TAZ1 causes premature degeneration of tapetum and pollen abortion in petunia. Plant Cell 14, 2353-2367. doi: 10.1105/tpc. 003061

Kuang, Y. F., Kirchoff, B. K., and Liao, J. P. (2012). Presence of the protruding oncus is affected by anther dehiscence and acetolysis technique. Grana 51, 253-262. doi: 10.1080/00173134.2012.660984

Kuang, Y. F., and Liao, J. P. (2015). Calcium distribution during pollen development of Uncaria hirsuta Havil. (Rubiaceae), with special reference to the function of the protruding oncus. Grana 54, 12-25. doi: 10.1080/00173134. 2014.962079

Li, H., Yuan, Z., Vizcay-Barrena, G., Yang, C. Y., Liang, W. Q., Zong, J., et al. (2011). PERSISTENT TAPETAL CELL1 encodes a PHD-finger protein that is required for tapetal cell death and pollen development in rice. Plant Physiol. 156, 615-630. doi: 10.1104/pp.111.175760

Li, N., Zhang, D. S., Liu, H. S., Yin, C. S., Li, X. X., Liang, W. Q., et al. (2006). The rice tapetum degeneration retardation gene is required for tapetum degradation and anther development. Plant Cell 18, 2999-3014. doi: 10.1105/tpc.106. 044107

Logacheva, M. D., Kasianov, A. S., Vinogradov, D. V., Samigullin, T. H., Gelfand, M. S., Makeev, V. J., et al. (2011). De novo sequencing and characterization of floral transcriptome in two species of buckwheat (Fagopyrum). BMC Genomics 12: 30. doi: 10.1186/1471-2164-12-30

Loraine, A. E., McCormick, S., Estrada, A., Patel, K., and Qin, P. (2013). RNA-Seq of Arabidopsis pollen uncovers novel transcription and alternative splicing. Plant Physiol. 162, 1092-1109. doi: 10.1104/pp.112.211441

Lu, L., Wortley, A. H., Li, D. Z., Wang, H., and Blackmore, S. (2015). Evolution of angiosperm pollen. 2. The basal angiosperms. Ann. Mo. Bot. Gard. 100, 227-269. doi: 10.3417/2012048

Luo, H., Lee, J. Y., Hu, Q., Nelson-Vasilchik, K., Eitas, T. K., Lickwar, C., et al. (2006). RTS, a rice anther-specific gene is required for male fertility and its promoter sequence directs tissue-specific gene expression in different plant species. Plant Mol. Biol. 62, 397-408. doi: 10.1007/s11103-0069031-0

Ma, J., Skibbe, D. S., Fernandes, J., and Walbot, V. (2008). Male reproductive development: gene expression profiling of maize anther and pollen ontogeny. Genome Biol. 9: R181. doi: 10.1186/gb-2008-9-12-r181

McCormick, S. (1993). Male gametophyte development. Plant Cell 5, 1265-1275. doi: $10.1105 /$ tpc.5.10.1265

Morant, M., Jorgensen, K., Schaller, H., Pinot, F., Lindberg Moller, B., WerckReichhart, D., et al. (2007). CYP703 is an ancient cytochrome P450 in land plants catalyzing in-chain hydroxylation of lauric acid to provide building blocks for sporopollenin synthesis in pollen. Plant Cell 19, 1473-1487. doi: 10.1105/tpc.106.045948

Ness, R. W., Siol, M., and Barrett, S. C. (2011). De novo sequence assembly and characterization of the floral transcriptome in cross- and self-fertilizing plants. BMC Genomics 12: 298. doi: 10.1186/1471-2164-12-298

Owen, H. A., and Makaroff, C. A. (1995). Ultrastructure of microsporogenesis and microgametogenesis in Arabidopsis thaliana (L). Heynh. ecotype Wassilewskija (Brassicaceae). Protoplasma 185, 7-21. doi: 10.1007/BF01272749

Pacini, E. (1997). Tapetum character states: analytical keys for tapetum types and activities. Can. J. Bot. 75, 1448-1459. doi: 10.1139/b97-859

Parchman, T. L., Geist, K. S., Grahnen, J. A., Benkman, C. W., and Buerkle, C. A. (2010). Transcriptome sequencing in an ecologically important tree species: assembly, annotation, and marker discovery. BMC Genomics 11: 180. doi: 10. 1186/1471-2164-11-180

Pina, C., Pinto, F., Feijo, J. A., and Becker, J. D. (2005). Gene family analysis of the Arabidopsis pollen transcriptome reveals biological implications for cell growth, division control, and gene expression regulation. Plant Physiol. 138, 744-756. doi: $10.1104 /$ pp.104.057935
Rotman, N., Durbarry, A., Wardle, A., Yang, W. C., Chaboud, A., Faure, J., et al. (2005). A novel class of MYB factors controls sperm-cell formation in plants. Curr. Biol. 15, 244-248. doi: 10.1016/j.cub.2005.01.013

Rutley, N., and Twell, D. (2015). A decade of pollen transcriptomics. Plant Reprod. 28, 73-89. doi: 10.1007/s00497-015-0261-7

Saldanha, A. J. (2004). Java Treeview-extensible visualization of microarray data. Bioinformatics 20, 3246-3248. doi: 10.1093/bioinformatics/bth349

Sanders, P. M., Bui, A. Q., Weterings, K., McIntire, K. N., Hsu, Y. C., Lee, P. Y., et al. (1999). Anther developmental defects in Arabidopsis thaliana male-sterile mutants. Sex. Plant Reprod. 11, 297-322. doi: 10.1007/s004970050158

Schnurr, J. A., Storey, K. K., Jung, H. J. G., Somers, D. A., and Gronwald, J. W. (2006). UDP-sugar pyrophosphorylase is essential for pollen development in Arabidopsis. Planta 224, 520-532. doi: 10.1007/s00425-006-0240-1

Scott, R. J., Spielman, M., and Dickinson, H. G. (2004). Stamen structure and function. Plant Cell 16, S46-S60. doi: 10.1105/tpc.017012

Shi, C. Y., Yang, H., Wei, C. L., Yu, O., Zhang, Z. Z., Jiang, C. J., et al. (2011). Deep sequencing of the Camellia sinensis transcriptome revealed candidate genes for major metabolic pathways of TET-specific compounds. BMC Genomics 12: 131. doi: 10.1186/1471-2164-12-131

Shi, J. X., Cui, M. H., Yang, L., Kim, Y. J., and Zhang, D. B. (2015). Genetic and biochemical mechanisms of pollen wall development. Trends Plant Sci. 20, 741-753. doi: 10.1016/j.tplants.2015.07.010

Sorensen, A. M., Krober, S., Unte, U. S., Huijser, P., Dekker, K., and Saedler, H. (2003). The Arabidopsis ABORTED MICROSPORES (AMS) gene encodes a MYC class transcription factor. Plant J. 33, 413-423. doi: 10.1046/j.1365-313X. 2003.01644.x

Souza, C. D. A., Kim, S. S., Koch, S., Kienow, L., Schneider, K., McKim, S. M., et al. (2009). A novel fatty Acyl-CoA synthetase is required for pollen development and sporopollenin biosynthesis in Arabidopsis. Plant Cell 21, 507-525. doi: $10.1105 /$ tpc. 108.062513

Tsuchiya, T., Toriyama, K., Ejiri, S., and Hinata, K. (1994). Molecular characterization of rice genes specifically expressed in the anther tapetum. Plant Mol. Biol. 26, 1737-1746. doi: 10.1007/BF00019488

Twell, D. (2011). Male gametogenesis and germline specification in flowering plants. Sex. Plant Reprod. 24, 149-160. doi: 10.1007/s00497-010-0157-5

Verelst, W., Saedler, H., and Münster, T. (2007a). MIKC* MADS-protein complexes bind motifs enriched in the proximal region of late-pollen-specific Arabidopsis promoters. Plant Physiol. 143, 447-460. doi: 10.1104/pp.106.089805

Verelst, W., Twell, D., de Folter, S., Immink, R., Saedler, H., and Münster, T. (2007b). MADS-complexes regulate transcriptome dynamics during pollen maturation. Genome Biol. 8: R249. doi: 10.1186/gb-2007-8-11-r249

Verstraete, B., Moon, H. K., Smets, E., and Huysmans, S. (2014). Orbicules in flowering plants: a phylogenetic perspective on their form and function. Bot. Rev. 80, 107-134. doi: 10.1007/s12229-014-9135-1

Vinckier, S., and Smets, E. (2002). Morphological and ultrastructural diversity of orbicules in relation to evolutionary tendencies in Apocynaceae s.l. Ann. Bot. 90, 647-662. doi: 10.1093/aob/mcf243

Vinckier, S., and Smets, E. (2003). Morphological and ultrastructural diversity of orbicules in Gentianaceae. Ann. Bot. 92, 657-672. doi: 10.1093/aob/mcg187

Vizcay-Barrena, G., and Wilson, Z. A. (2006). Altered tapetal PCD and pollen wall development in the Arabidopsis ms1 mutant. J. Exp. Bot. 57, 2709-2717. doi: $10.1093 /$ jxb/erl032

Wan, L. L., Zha, W. J., Cheng, X. Y., Liu, C., Lv, L., Liu, C. X., et al. (2011). A rice beta-1,3-glucanase gene Osg1 is required for callose degradation in pollen development. Planta 233, 309-323. doi: 10.1007/s00425-010-1301-z

Wang, A. M., Xia, Q., Xie, W. S., Datla, R., and Selvaraj, G. (2003). The classical Ubisch bodies carry a sporophytically produced structural protein (RAFTIN) that is essential for pollen development. Proc. Natl. Acad. Sci. U.S.A. 100, 14487-14492. doi: 10.1073/pnas.2231254100

Wang, G., Du, X., Ji, J., Guan, C., Li, Z., and Josine, T. L. (2015). De novo characterization of the Lycium chinense Mill. leaf transcriptome and analysis of candidate genes involved in carotenoid biosynthesis. Gene 555, 458-463. doi: 10.1016/j.gene.2014.10.058

Wang, Z., Gerstein, M., and Snyder, M. (2009). RNA-Seq: a revolutionary tool for transcriptomics. Nat. Rev. Genet. 10, 57-63. doi: 10.1038/nrg2484

Wang, Z. Y., Fang, B. P., Chen, J. Y., Zhang, X. J., Luo, Z. X., Huang, L. F., et al. (2010). De novo assembly and characterization of root transcriptome using Illumina paired-end sequencing and development of cSSR markers in sweet 
potato (Ipomoea batatas). BMC Genomics 11: 726. doi: 10.1186/1471-216411-726

Wei, L. Q., Xu, W. Y., Deng, Z. Y., Su, Z., Xue, Y., and Wang, T. (2010). Genomescale analysis and comparison of gene expression profiles in developing and germinated pollen in Oryza sativa. BMC Genomics 11: 338. doi: 10.1186/14712164-11-338

Wilson, Z. A., and Zhang, D. B. (2009). From Arabidopsis to rice: pathways in pollen development. J. Exp. Bot. 60, 1479-1492. doi: 10.1093/jxb/erp095

Wortley, A. H., Wang, H., Lu, L., Li, D. Z., and Blackmore, S. (2015). Evolution of angiosperm pollen. 1. Introduction. Ann. Mo. Bot. Gard. 100, 177-226. doi: 10.3417/2012047

Xu, J., Ding, Z., Vizcay-Barrena, G., Shi, J. X., Liang, W. Q., Yuan, Z., et al. (2014). ABORTED MICROSPORES acts as a master regulator of pollen wall formation in Arabidopsis. Plant Cell 26, 1544-1556. doi: 10.1105/tpc.114. 122986

Xu, J., Yang, C. Y., Yuan, Z., Zhang, D. S., Gondwe, M. Y., Ding, Z. W., et al. (2010). The ABORTED MICROSPORES regulatory network is required for postmeiotic male reproductive development in Arabidopsis thaliana. Plant Cell 22, 91-107. doi: 10.1105/tpc.109.071803

Yang, S. L., Xie, L. F., Mao, H. Z., Puah, C. S., Yang, W. C., Jiang, L., et al. (2003). The TAPETUM DETERMINANT1 gene is required for cell specialization in the Arabidopsis anther. Plant Cell 15, 2792-2804. doi: 10.1105/tpc.016618

Ye, J., Fang, L., Zheng, H. K., Zhang, Y., Chen, J., Zhang, Z. J., et al. (2006). WEGO: a web tool for plotting GO annotations. Nucleic Acids Res. 34, W293-W297. doi: 10.1093/nar/gkl031

Zhang, D. B., Luo, X., and Zhu, L. (2011). Cytological analysis and genetic control of rice anther development. J. Genet. Genomics 38, 379-390. doi: 10.1016/j.jgg. 2011.08.001

Zhang, D. B., and Wilson, Z. A. (2009). Stamen specification and anther development in rice. Chinese Sci. Bull. 54, 2342-2353. doi: 10.1007/s11434-0090348-3

Zhang, D. D., Liu, D., Lv, X. M., Wang, Y., Xun, Z. L., Liu, Z. X., et al. (2014). The cysteine protease CEP1, a key executor involved in tapetal programmed cell death, regulates pollen development in Arabidopsis. Plant Cell 26, 2939-2961. doi: $10.1105 /$ tpc.114.127282

Zhang, D. S., Liang, W. Q., Yin, C. S., Zong, J., Gu, F. W., and Zhang, D. B. (2010). OsC6, encoding a lipid transfer protein, is required for postmeiotic anther development in rice. Plant Physiol. 154, 149-162. doi: 10.1104/pp.110. 15886
Zhang, F. J., Wang, Z. Q., Dong, W., Sun, C. Q., Wang, H. B., Song, A. P., et al. (2014). Transcriptomic and proteomic analysis reveals mechanisms of embryo abortion during chrysanthemum cross breeding. Sci. Rep. 4: 6536. doi: 10.1038/srep06536

Zhang, W., Sun, Y. J., Timofejeva, L., Chen, C. B., Grossniklaus, U., and Ma, H. (2006). Regulation of Arabidopsis tapetum development and function by dysfunctional tapetum1 (dyt1) encoding a putative bHLH transcription factor. Development 133, 3085-3095. doi: 10.1242/dev.02463

Zhang, X. M., Zhao, L., Larson-Rabin, Z., Li, D. Z., and Guo, Z. H. (2012). De novo sequencing and characterization of the floral transcriptome of Dendrocalamus latiflorus (Poaceae : Bambusoideae). PLoS ONE 7:e42082. doi: 10.1371/journal. pone.0042082

Zhang, Z. B., Zhu, J., Gao, J. F., Wang, C., Li, H., Li, H., et al. (2007). Transcription factor AtMYB103 is required for anther development by regulating tapetum development, callose dissolution and exine formation in Arabidopsis. Plant J. 52, 528-538. doi: 10.1111/j.1365-313X.2007.03254.x

Zhao, D. Z., Wang, G. F., Speal, B., and Ma, H. (2002). The EXCESS MICROSPOROCYTES1 gene encodes a putative leucine-rich repeat receptor protein kinase that controls somatic and reproductive cell fates in the Arabidopsis anther. Gene. Dev. 16, 2021-2031. doi: 10.1101/gad.997902

Zhao, S. R., Fung-Leung, W.-P., Bittner, A., Ngo, K., and Liu, X. J. (2014). Comparison of RNA-Seq and microarray in transcriptome profiling of activated $\mathrm{T}$ cells. PLoS ONE 9: e78644. doi: 10.1371/journal.pone. 0078644

Zhu, E. G., You, C. J., Wang, S. S., Cui, J., Niu, B. X., Wang, Y. X., et al. (2015). The DYT1-interacting proteins bHLH010, bHLH089 and bHLH091 are redundantly required for Arabidopsis anther development and transcriptome. Plant J. 83, 976-990. doi: 10.1111/tpj.12942

Conflict of Interest Statement: The authors declare that the research was conducted in the absence of any commercial or financial relationships that could be construed as a potential conflict of interest.

Copyright (c) 2017 Yue, Twell, Kuang, Liao and Zhou. This is an open-access article distributed under the terms of the Creative Commons Attribution License (CC BY). The use, distribution or reproduction in other forums is permitted, provided the original author(s) or licensor are credited and that the original publication in this journal is cited, in accordance with accepted academic practice. No use, distribution or reproduction is permitted which does not comply with these terms. 